\title{
Inversion-Vibration and Inversion-Rotation Interactions in the Ammonia Molecule*
}

\author{
William T. Weeks, $†$ Karl T. Hecht, and David M. Dexisox \\ Harrison M. Randall Laboratory of Physics, The Lniversity of Michigan. \\ Ann Arbor, Michigan
}

\begin{abstract}
An at tempt has been made to extend the theory of ammonia inversion in order to account for the dependence of the inversion splitting on the full set of vibrational and rotational quantum numbers. The potential energy of ammonia is approximated by a double minimum potential $V^{r}(\zeta)$ plus the potential of a system of harmonic oscillators in the remaining five vibrational coordi. nates. $V(\zeta)$ has been chosen to have the form $V(\zeta)=-2 F \cos (\zeta / \mathrm{L})+2 G \cos$ $(2 \zeta / L)$ in which $\zeta$ is an inversion coordinate and $L$ a constant $(\zeta \leqq \pi L$. The double minimum wave functions are computed numerically. Inversionvibration interactions are obtained by developing the parameters $F$ and $G$. which are regarded as mild functions of the five vibrational coordinates, in a Taylor expansion in the vibrational coordinates. With the exception of the state $2 \nu_{4}{ }^{0}$ this potential aceounts for the dependence of the inversion splittings on the vibrational quantum numbers of the two doubly degenerate modes $\nu:$ and $\nu_{4}$ (eleven experimental data are fitted with four empirical interaction constants). However, the potential fails to deseribe completely the intertation between the inversion coordinate and the remaining nondegenerate vibritional coordinate associated with $\nu_{1}$. Since the task of diagonalizing the complete rotation-inversion Hamiltonian is complicated by the presence of several resonances, the rotation-inversion constant. $B^{-}-B^{+}$and $C^{\prime-\cdots}-C^{+}$are calculated only from the lowest order vibration-rotation-inversion Hamiltonian. The calculated constants for the pure inversion states $n_{2}=0,1,2$, and 3 and the states $n_{2}=1$ in combination with the remaining vibrational modes agree surprisingly well with the experimentally observed values.
\end{abstract}

\section{INTROIDUCTION}

Recent investigations of the ammonia spectrum by Benedict ot al. (1-1) and by Craring et al. (5) have uncovered at wealth of new data revealing the dependence of the inversion splitting on the complete set of vibrational and rotational quantum numbers. In the past, theories of ammonia inversion have aimed at predieting the dependence of the splitting on the guantum number n: asso-

* Supported in part by the Office of Naval Research under Navy Theoretical Physics Contract No. Nonr' 1224(15).

$\dagger$ Present address: Data Systems Division, International Business Machines, Pough keepsie, New York. Dow Chemical Company Fellow 1956-7, Walter Lewis Fellow 1957-8. Lniversity of Michigan Fellow 1958-9. 
TABLE I

INversion Splitting in $\mathrm{NH}_{3}\left(\mathrm{~cm}^{-1}\right)$

\begin{tabular}{|c|c|c|c|}
\hline$n_{1} n_{2^{ \pm}} n_{3}{ }^{l}{ }_{3} n_{1}{ }^{l}{ }^{2}$ & $\Delta_{n}{ }^{0}$ & $B_{n}^{--}-B_{n}^{+}$ & $C_{n}^{-}-C_{n}^{+}$ \\
\hline $\begin{array}{llll}0 & 0 & 0^{0} & 0^{\circ}\end{array}$ & 0.793 & -0.005054 & 0.001998 \\
\hline $010^{0} 0^{0}$ & 35.81 & -0.1817 & 0.0721 \\
\hline $020^{0} 0^{0}$ & 284.74 & -0.535 & 0.231 \\
\hline $030^{\circ} 0^{\circ}$ & 512.02 & -0.3041 & 0.1034 \\
\hline $\begin{array}{lllll}0 & 0 & 1^{1} & 0^{\circ}\end{array}$ & 0.35 & -0.0036 & 0.0007 \\
\hline $002^{2} 0^{4}$ & 0.43 & & \\
\hline $\begin{array}{llll}0 & 0 & 0^{0} & 1^{1}\end{array}$ & 1.01 & 0.048 & 0.011 \\
\hline $000^{0} 2^{0}$ & 2.24 & & \\
\hline $\begin{array}{llll}0 & 0 & 0^{9} & 2^{2}\end{array}$ & 1.42 & & \\
\hline $001^{1} 1^{1}$ & $0.5 i$ & & \\
\hline $100^{0} 0^{0}$ & 0.99 & -0.012 & 0.003 \\
\hline $100^{\circ} 1^{1}$ & 0.86 & & \\
\hline $011^{1} 0^{9}$ & 18.49 & -0.0984 & 0.0429 \\
\hline $\begin{array}{llll}0 & 1 & 0^{0} & 1^{i}\end{array}$ & 45.4 & -0.191 & 0.097 \\
\hline $\begin{array}{llll}0 & 1 & 1^{1} & 1^{1}\end{array}$ & 23.68 & -0.130 & 0.054 \\
\hline $110^{0} 0^{0}$ & 25.55 & -0.1265 & 0.0470 \\
\hline
\end{tabular}

ciated with the mode in which the pyramid height changes most drastically (6-9). In this paper an attempt is made to extend the theory to include the dependence of the inversion splitting on the full set of vibrational and rotational quantum numbers $n_{1}, n_{2}^{ \pm}, n_{3}^{l_{3}}, n_{4}^{l_{4}}, J$, and $K$, where the designation of quantum numbers is that of Benedict et al. (1).

The currently available data on the inversion splitting in $\mathrm{NH}_{3}$ are summarized in Table I. Although the inversion splitting depends mainly on the quantum number $n_{2}$, it is clear that it also exhibits a significant dependence on the remaining vibrational quantum numbers $n_{1}, n_{3}^{l_{3}}$, and $n_{4}^{l_{4}}$. This leads immediately to the conclusion that there must exist interactions between the inversion coordinate and the remaining vibrational coordinates. In the present paper a scheme is proposed for describing and evaluating these interactions. The potential energy function for ammonia is represented, in lowest approximation, by a double minimum potential involving the inversion coordinate plus the potential of a system of uncoupled harmonic oscillators involving the five remaining vibrational coordinates. Interactions are obtained by interpreting the parameters occurring in the double minimum potential as mild functions of the remaining vibrational coordinates through the use of a Taylor series expansion. In a similar fashion the vibration-rotation interactions affecting the inversion splitting have

${ }^{1}$ By treating the remaining degrees of vibrational freedom by means of uncoupled harmonic oscillators, the usual anharmonicities involving only these degrees of freedom are ignored. Although these are important for a complete description of the spectrum it is felt that they will not contribute significantly to the inversion splitting. 
been introduced and calculated. These lead to numerical values which can be compared with the experimental quantities $B^{-}-B^{+}$and $C^{-}-C^{+}$.

\section{INTERNAL COORDINATES AND THE VIBRATIONAL KINETIC:}

\section{ENERGY}

As a method of specifying a set of internal coordinates for ammonia it is convenient to start with a Cartesian reference frame fixed in space and with origin at the center of mass of the molecule as shown in Fig. 1. Let $x_{i}, y_{i}$, and $z_{i}$ be the Cartesian coordinates of the $i$ th atom with respect to this reference frame. The subseripts $i=1,2$, and 3 refer to hydrogen atoms and $i=4$ to the nitrogen atom. In either of the two equilibrium configurations of the molesule the nitrogen atom lies on the $z$-axis and the number three hydrogen atom is chosen to lie in the $y-z$ plane. The twelve Cartesian coordinates of the four atoms can he replaced by six internal coordinates $u_{2}(i=1, \cdots(i)$ by means of the following seheme where $m$ is the mass of a hydrogen atom and $M$ the mass of the nitrogen atom.

$$
\begin{aligned}
& x_{1}=-\frac{\sqrt{3}}{2} u_{1}-\frac{\sqrt{3}}{2} u_{3}-\frac{1}{2} u_{4}-\frac{M}{M+3 m} u_{6}, \\
& y_{1}=-\frac{1}{2} u_{1}+\frac{1}{2} u_{3}-\frac{\sqrt{3}}{2} u_{4}-\frac{M}{M+3} \frac{M}{M} u_{5}, \\
& z_{1}=-\frac{M}{M+3 m}\left[u_{2}+\left(\frac{u_{2}}{u_{1}}\right) u_{5}+\sqrt{3}\left(\frac{u_{2}}{u_{1}}\right) u_{6}\right] \text {, } \\
& x_{2}=\frac{\sqrt{3}}{2} u_{1}+\frac{\sqrt{3}}{2} u_{3}-\frac{1}{2} u_{4}-\frac{M}{M+3 m} u_{6}, \\
& y_{2}=-\frac{1}{2} u_{1}+\frac{1}{2} u_{3}+\frac{\sqrt{3}}{2} u_{4}-\frac{M}{M+3 m} u_{5} \text {, } \\
& z_{2}=-\frac{M}{M+3 m}\left[u_{2}+\left(\frac{u_{2}}{u_{1}}\right) u_{5}-\sqrt{3}\left(\frac{u_{2}}{u_{1}}\right) u_{6}\right] \text {, } \\
& x_{3}=u_{4}-\frac{M}{M+3 m} u_{6} \\
& y_{3}=u_{1}-u_{3}-\frac{M}{M+3 m} u_{5} \text {, } \\
& z_{3}=-\frac{M}{M+3 m}\left[u_{2}-2\left(\frac{u_{2}}{u_{1}}\right) u_{5}\right] \text {. } \\
& x_{4}=\frac{3 m}{M+3 m} u_{c} \\
& y_{4}=\frac{3 m}{M+3 m} u_{5} \\
& z_{4}=\frac{3 m}{M+3 m} u_{2} \text {. }
\end{aligned}
$$



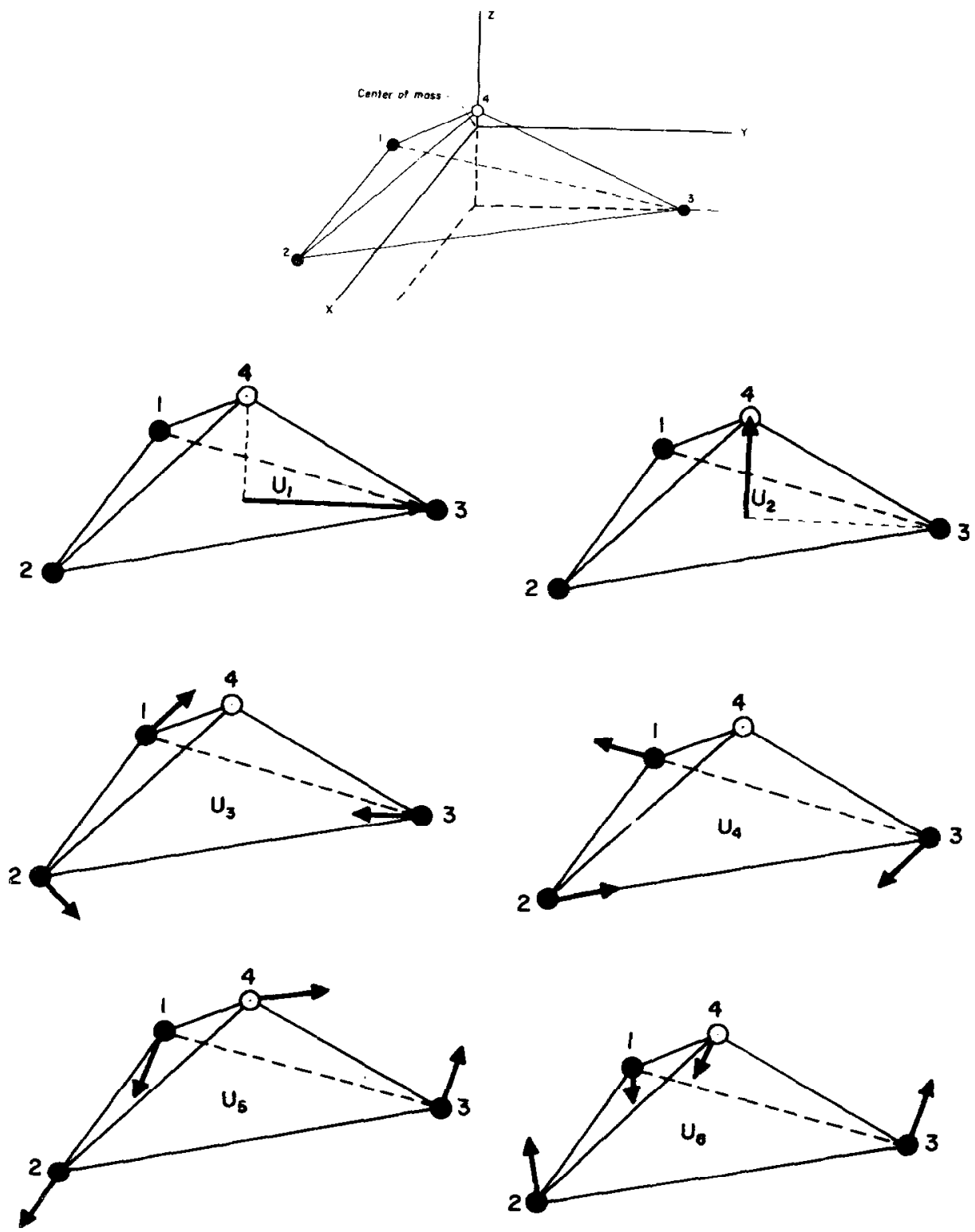

FI(x. 1. The internal coordinates

To discover the physical significance of these coordinates consider first the case where $u_{3}=u_{4}=u_{5}=u_{6}=0$. It is apparent from the equations that $u_{2}$ is rqual to the height of the ammonia pyramid and $u_{1}$ equals the distance from a hydrogen atom to the center of the hydrogen triangle. All configurations that 
arise when $u_{3}=u_{4}=u_{5}=u_{6}=0$ have pyramidal symmetry. That is, the hydrogen atoms form an equilateral triangle and the three $\mathrm{N}-\mathrm{H}$ bond lengths are equal. On the other hand, the coordinates $u_{3}, u_{4}, u_{5}$, and $u_{6}$ relate to deviations from pyramidal symmetry. The coordinates $u_{3}$ and $u_{4}$ describe a configuration in which the nitrogen atom remains stationary and the hydrogen atoms undergo displacements parallel to the $x-y$ plane. $u_{5}$, however, involves a displarement of the nileogen atom parallel to the $y$-axis with a corresponding tipping of the hydrogen triangle while $u_{6}$ represents a displacement of the nitrogen atom parallel to the $x$-axis again with a tipping of the hydrogen triangle. For both $u_{5}$ and $u_{6}$ all the $\mathrm{H}-\mathrm{H}$ distances remain invariant.

The two equilibrium configurations are given by

$$
u_{1}=u_{1}^{\prime \prime}, \quad u_{2}= \pm u_{2}{ }^{n}, \quad u_{3}=u_{4}=u_{5}=u_{6}=0,
$$

wherewhere $u_{1}^{\prime \prime}$ and $u_{2}^{\prime \prime}$ are approximately $0.94 \times 10^{-8}$ and $0.38 \times 10^{-x} \mathrm{~cm}$, respectively. Inversion of the molecule through a plane passing through the center of mass and perpendicular to the symmetry axis is accomplished by means of the transformation

$$
u_{2} \rightarrow-u_{2}, \quad u_{i} \rightarrow u_{i} \quad(i \neq 2)
$$

The internal coordinates have been chosen in such a way that for all values of the coordinates and their time derivatives the linear momentum of the molecule: remains zero. The angular momentum also remains exactly zero for all motions in which the molecule retains pyramidal symmetry, that is, where $u_{3}-u_{4}-$ $u_{5}=u_{6}=0$, but for all values of $u_{1}$ and $u_{2}$. For those motions however in which the pyramidal symmetry is destroyed, there will exist small terms in the angular momentum.

A simple calculation yields the vibrational kinetic energy

$$
\begin{aligned}
T_{v i b}=\frac{1}{2}\left\{3 m \dot{u}_{1}{ }^{2}\right. & +\mu \dot{u}_{2}{ }^{2}+3 m\left(\dot{u}_{3}{ }^{2}+\dot{u}_{4}{ }^{2}\right) \\
& +\mu \rho\left(u_{1}, u_{2}\right)\left(\dot{u}_{5}{ }^{2}+\dot{u}_{6}{ }^{2}\right)+\frac{4 \mu^{2}}{3 m}\left(u_{5} \dot{u}_{5}+u_{6} \dot{u}_{6}\right) \frac{u_{2}}{u_{1}} \frac{d}{d t}\left(\frac{u_{2}}{u_{1}}\right) \\
& \left.+\frac{2 \mu^{2}}{3 m}\left(u_{5}{ }^{2}+u_{6}{ }^{2}\right)\left[\frac{d}{d t}\left(\frac{u_{2}}{u_{1}}\right)\right]^{2}\right\},
\end{aligned}
$$

where $\mu=3 m M / M+3 m$ and $\rho\left(u_{1}, u_{2}\right)=\left[1+(2 \mu / 3 m)\left(u_{2} / u_{1}\right)^{2}\right]$. Thes symmetry of the coordinates is such that the leading terms in the Taylor expansion of the potential function about an equilibrium configuration would be

$$
\begin{aligned}
V= & 1_{2}\left\{3 b\left(u_{1}-u_{1}{ }^{0}\right)^{2}+2 \sqrt{3} c\left(u_{1}-u_{1}{ }^{0}\right)\left(u_{2}-u_{2}{ }^{0}\right)+a\left(u_{2}-u_{2}{ }^{0}\right)^{2}\right. \\
& \left.+3 \beta\left(u_{3}{ }^{2}+u_{4}{ }^{2}\right)+2 \sqrt{3} \gamma \rho_{0}\left(u_{3} u_{5}+u_{4} u_{6}\right)+\alpha \rho_{0}{ }^{2}\left(u_{5}{ }^{2}+u_{6}{ }^{2}\right)+\cdots\right\}
\end{aligned}
$$


where $\rho_{0}=\left[1+(2 \mu / 3 m)\left(u_{2}^{0} / u_{1}^{0}\right)^{2}\right]$, and where the force constants $a, b, c, \alpha, \beta$, and $\gamma$, defined previously by Dennison (10), have been used.

It will now prove convenient to replace the coordinates $u_{3}, u_{4}, u_{5}$, and $u_{6}$ by a new set of internal coordinates $Q_{3 x}, Q_{3 y}, Q_{4 x}$, and $Q_{4 y}$ defined by

$$
\begin{aligned}
& Q_{3 x}=\cos \tau(3 m)^{1 / 2} u_{4}+\sin \tau\left[\mu \rho\left(u_{1}, u_{2}\right)\right]^{1 / 2} u_{6}, \\
& Q_{4 x}=-\sin \tau(3 m)^{1 / 2} u_{4}+\cos \tau\left[u \rho\left(u_{1}, u_{2}\right)\right]^{1 / 2} u_{6}, \\
& Q_{3 y}=\cos \tau(3 m)^{1 / 2} u_{3}+\sin \tau\left[\mu \rho\left(u_{1}, u_{2}\right)\right]^{1 / 2} u_{5}, \\
& Q_{4 y}=-\sin \tau(3 m)^{1 / 2} u_{3}+\cos \tau\left[\mu \rho\left(u_{1}, u_{2}\right)\right]^{1 / 2} u_{5},
\end{aligned}
$$

where

$$
\cos 2_{\tau}=\left(\frac{\beta}{m}-\frac{\alpha \rho_{0}}{\mu}\right)\left[\left(\frac{\beta}{m}-\frac{\alpha \rho_{0}}{\mu}\right)^{2}+\frac{4 \gamma^{2} \rho_{0}}{m \mu}\right]^{-1 / 2} .
$$

The coordinates $Q_{3 x}, Q_{3 y}, Q_{4 x}$, and $Q_{4 y}$ are defined in such a way that, in the vicinity of the equilibrium configurations, they become the normal coordinates describing the perpendicular vibrations. Note, however, that in general they depend on the two axially symmetric coordinates $u_{1}$ and $u_{2}$. Finally, the coordinates $u_{1}$ and $u_{2}$ are replaced by a pair of coordinates $\sigma$ and $\zeta$ defined by

$$
\begin{aligned}
(3 m)^{1 / 2} u_{1} & =a_{0} \sinh \sigma \cos \zeta, \\
(\mu)^{1 / 2} u_{2} & =a_{0} \cosh \sigma \sin \zeta,
\end{aligned}
$$

where $a_{0}^{2}=\mu\left(u_{2}^{0}\right)^{2}-3 m\left(u_{1}^{0}\right)^{2}+\sqrt{3} u_{1}^{0} u_{2}{ }^{0}(\mu b-m a) / c$ and $\sigma \geqq 0 ;-\pi / 2 \leqq$ $\zeta \leqq \pi / 2$. The constant $a_{0}$ has been chosen so that in the neighborhood of an equilibrium configuration $\delta \sigma=\sigma-\sigma_{0}$ and $\delta \zeta=\zeta-\zeta_{0}$ are proportional to the two symmetric normal coordinates of ammonia. In fact, it is easy to show that this choice of $a_{0}$ leads to the relations

$$
1 / 2\left(3 m \dot{u}_{1}^{2}+\mu \dot{u}_{2}^{2}\right)=1 / 2 a_{0}^{2}\left(\cosh ^{2} \sigma_{0}-\sin ^{2} \zeta_{0}\right)\left(\delta \dot{\sigma}^{2}+\delta \dot{\zeta}^{2}\right)
$$

and

$$
\begin{aligned}
3 / 2 b\left(u_{1}-u_{1}^{0}\right)^{2}+\sqrt{ } 3 c\left(u_{1}-u_{1}^{0}\right) & \left(u_{2}-u_{2}^{0}\right)+1 / 2 a\left(u_{2}-u_{2}{ }^{0}\right)^{2} \\
& =1 / 2 a_{0}{ }^{2}\left(\cosh ^{2} \sigma_{0}-\sin ^{2} \zeta_{0}\right)\left(\lambda_{1} \delta \sigma^{2}+\lambda_{2} \delta \xi^{2}\right),
\end{aligned}
$$

where

$$
\left.\begin{array}{l}
\lambda_{1} \\
\lambda_{2}
\end{array}\right\}=\frac{1}{2}\left(\frac{b}{m}+\frac{a}{\mu}\right) \pm\left[\frac{1}{4}\left(\frac{b}{m}-\frac{a}{\mu}\right)^{2}+\frac{c^{2}}{\mu m}\right]^{1 / 2} .
$$

In these equations $\sigma_{0}$ and $\pm \zeta_{0}$ are the equilibrium values of $\sigma$ and $\zeta$ and describe the two equilibrium configurations of the molecule. The physical significance of $\sigma$ and $\zeta$ is best seen by inverting $\mathrm{Eq}$. (6). It will be recalled that $u_{2}$ is the height of the ammonia pyramid and $u_{1}$ is the distance from a hydrogen atom to the 


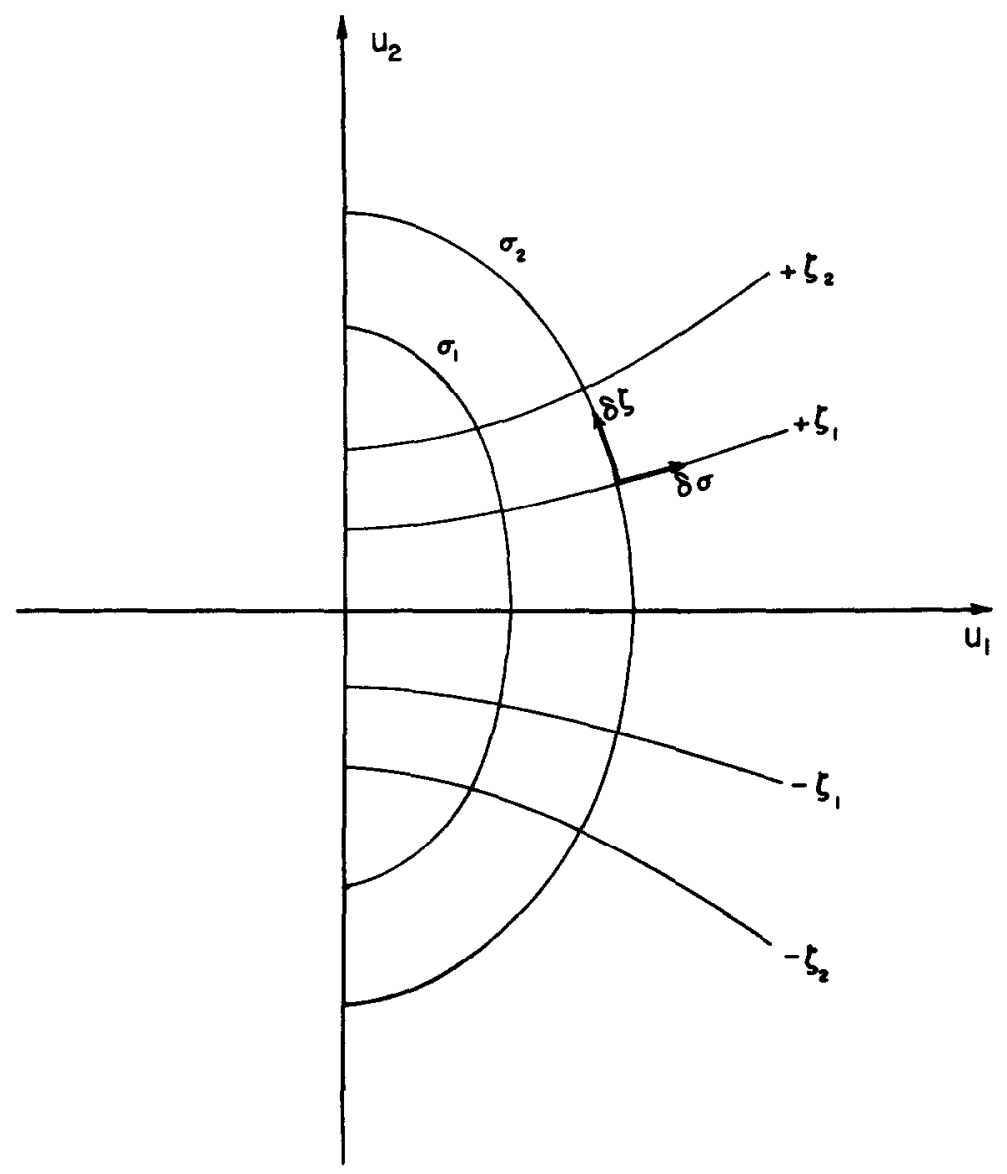

Fici. 2. The coordinates $\sigma$ and $\xi$

center of the hydrogen triangle. One finds that the family of curves in the $u_{1}-$ $u_{2}$ plane with $\sigma$ constant are ellipses and the family of curves with $\zeta$ constant are hyperbolas. This is illustrated in lig. 2. The coordinate $\zeta$ may be identified as an inversion coordinate since the molecule ean be inverted from one equilibrium configuration to the other by changing $\zeta$ continuously from $\zeta_{0}$ to $-\zeta_{0}$ while keeping $\sigma$ fixed at its equilibrium value $\sigma_{0}$.

Upon introducing the coordinates $\sigma, \zeta, Q_{3 x}, Q_{3 y}, Q_{4 x}$, and $Q_{4 y}$ into Fa. (2) one obtains for the vibrational kinetic energy

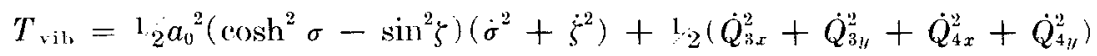

$$
\begin{aligned}
& +\left[\sin \tau \mathbf{R}_{3}+\cos \tau \mathbf{R}_{4}\right]^{2} \cdot\left[\begin{array}{l}
\cosh \sigma \sinh \sigma \zeta-\cos \zeta \sin \zeta \dot{\sigma} \\
\sinh ^{2} \sigma \cos ^{2} \zeta+\cosh ^{2} \sigma \sin ^{2} \zeta
\end{array}\right]^{2} \text {, }
\end{aligned}
$$


where $\mathbf{R}_{3}$ is a vector with components $Q_{3 x}, Q_{3 y}$, and $\mathbf{R}_{4}$ is a vector with components $Q_{4 x}, Q_{4 y}$. So far no approximations have been made and Eq. (9) is rigorous.

\section{THE POTENTIAL ENERGY AND THE VIBRATIONAL HAMILTONIAN}

The present state of development of molecular theory is not sufficiently advanced to allow an a priori derivation of a potential function for a molecule as complex as ammonia. The best that can be done is to choose a function with plausible physical characteristics. The function must of course satisfy the symmetry requirements and should contain a number of adjustable parameters which will be determined by comparing the results of the calculation with the experimental data.

It may be remarked that, in the six-dimensional coordinate space, there exists a path connecting the two equilibrium configurations for which the potential has the smallest set of values. It may be thought of as the lowest pass over the potential mountains standing in the way of inversion. A plausible choice for this path is that ellipse for which the coordinate $\zeta$ alone varies, all other coordinates remaining zero. The double minimum character of the potential will therefore be described through a suitable function of $\zeta$. The usual normal vibration properties of the molecule further require the presence of terms quadratic in the remaining coordinates. It will be assumed that these always remain near their equilibrium values, so that $\delta \sigma, Q_{3 x}, Q_{3 y}, Q_{4 x}$, and $Q_{4 y}$ are small quantities. In the subsequent discussion these small oscillation coordinates will be replaced by their dimensionless counterparts $q_{1}, q_{3 x}, q_{3 y}, q_{4 x}$, and $q_{4 y}$, defined by

$$
\begin{aligned}
\delta \sigma & =\left[h / 4 \pi^{2} c \omega_{1} a_{0}^{2}\left(\cosh ^{2} \sigma_{0}-\sin ^{2} \zeta_{0}\right)\right]^{1 / 2} q_{1}, \\
Q_{i \alpha} & =\left(h / 4 \pi^{2} c \omega_{i}\right)^{142} q_{i \alpha}, \quad i=3,4, \quad \alpha=x, y,
\end{aligned}
$$

where these reduce to (dimensionless) normal coordinates in the vicinity of either equilibrium configuration of the molecule.

The above considerations have therefore led to choosing a potential of the following form,

$$
V / h c=V_{0}(\zeta) / h c+1 / 2 c_{1} q_{1}^{2}+1 / 2 c_{3}\left(q_{3 x}^{2}+q_{3 y}^{2}\right)+1 / 2 c_{4}\left(q_{4 x}^{2}+q_{4 y}^{2}\right),
$$

where $c_{1}, c_{3}$, and $c_{4}$ are constants and $V_{0}(\zeta)$ is a double minimum potential with minima at $\zeta_{0}$ and $-\zeta_{0}$ and a central maximum as $\zeta=0$.

Although the inversion splitting is an extremely sensitive function of the inversion potential, the WKB treatment of ammonia inversion given by Dennison and Uhlenbeck ( 6 ) suggests that there may be many mathematical functions $V_{0}(\zeta)$ which can account, at least approximately, for the observed splitting. Practical considerations, however, limit the form of $V_{0}(\zeta)$ to those functions for which energy levels and wave functions can be found with reasonable ease. 
A possible candidate might be the potential proposed by Manning ( 7 ). Although the energy levels for this potential are relatively easy to find the wave fiunctions would be somewhat cumbersome to use in a perturbation calculation. In order to obtain more manageable wave functions, the double minimum potential

$$
\begin{array}{rlrl}
V_{0}(\zeta) / h c & =-2 F \cos (\zeta / L)+2 G \cos (2 \zeta / L) & \text { for }|\zeta| \leqq \pi L \\
& =2(F+G) \quad \text { for } \pi L<|\zeta| \leqq \pi 2
\end{array}
$$

will be used in the present investigation. In $\mathrm{E}_{1}$. (12), $F$ and $(r$ are positive ronstants such that $F \leqq 4(r$ and $L$ is a positive constant such that $L \leqq 1.2$. This potential has a central maximum at $\zeta=0$ and minima at $\zeta= \pm \zeta_{\text {" where }}$ $\cos \left(\zeta_{0} / L\right)=F / 4 G_{x}$. It has the general form to be expected of an inversion potential in the neighborhoods of $\zeta_{0}$ and $-\zeta_{0}$ and throughout the interval $-\zeta_{11}<$ $\zeta\left\langle\zeta_{0}\right.$. For $|\zeta| \gg\left|\zeta_{0}\right|$ it is undoubtedly a poor approximation to the true ammonia potential, but the low-lying energy levels shouod not be affected very much by the behavior of the potential at large values of $|\zeta|$.

Interactions between the inversion coordinate and the remaining vibrational roordinates are obtained by assuming that the parameters $F$ and $f$ are not true constants but mild functions of the coordinates $q_{1}, q_{3 x}, q_{3 y}, q_{4 x}$, and $q_{4 y}$. Nince these coordinates represent very small oscillations it should be possible to obtain a good approximation by expanding $F$ and $G$ in a Taylor series and retaining only the leading terms. In principle, $L$ could be expanded in a Taylor sories also. However, an expansion of $L, F$, and ( $r$ would yield more unknown constants than can be determined by the available data on ammonia. Thus, in prartice, one of the three parameters must be regarded as a genuine constant. The argument for rhoosing $L$ as the constant ran be stated as follows. The inversion spliting is expected to be a very sensitive function of the potential batrier height and the separation of the potential minima. The height of the eentral barrier for the potential given by $\mathrm{E}\left(\mathrm{q} .(12)\right.$ is $4\left(x\left(1-F / 4(t)^{2}\right.\right.$ and is independent. of $L$. Furthermore, the separation of the potential minima can be shown to be very insensitive to the value of $L$. Thus, if one of the three parameters must be regarded as a true constant then $L$ is probably the best choice.

The form of the expansion of $F$ and $G$ is restricted. First, the potential must. be invariant under the symmetry operations of the molecular point group. This excludes, for example, terms linear in the $q_{k \alpha}$ and $q_{4 \alpha}$. Sccondly, since the (o)ordinates $q_{1},\left(\zeta-\zeta_{0}\right), q_{3 x}, q_{3 y}, q_{4 x}$, and $q_{4 y}$ are proportional to normal coordinates in the vicinity of an equilibrium configuration, the full potential must sutisfy the equilibrium conditions $\left(\partial V / \partial q_{i}\right)_{0}=0$ and must contain no cross products in the quadratic development about an equilibrium configuration. The combination of these two requirements exceludes terms linear in $q_{1}$. linally, sinces $F$ and (f are assumed to be mild functions, only the first significant terms in the vibrational coordinates will be retained. Out to cuadratic terms the most general form of the expansion of $F$ and $G$ is 


$$
\begin{aligned}
& F=F_{0}+F_{1} q_{1}{ }^{2}+F_{3} r_{3}{ }^{2}+F_{4} r_{4}{ }^{2}+k \cos \left(2 \zeta_{0} / L\right) \mathrm{r}_{3} \cdot \mathrm{r}_{4}, \\
& G=G_{0}+G_{1} q_{1}{ }^{2}+G_{3} r_{3}{ }^{2}+G_{4} r_{4}{ }^{2}+k \cos \left(\zeta_{0} / L\right) \mathbf{r}_{3} \cdot \mathbf{r}_{4},
\end{aligned}
$$

where $F_{0}, F_{1}, F_{3}, F_{4}, G_{0}, G_{1}, G_{3}, G_{4}, L$, and $k$ are true constants. Here $\mathrm{r}_{3}$ and $\mathrm{r}_{4}$ are vectors with components $q_{3 x}, q_{3 y}$, and $q_{4 x}, q_{4 y}$, respectively. As a means of keeping the ensuing computations reasonably manageable the constant $k$ will be set equal to zero although no real physical justification can be given for doing so. Thus one obtains

$$
\begin{aligned}
V / h c=1 / 2 c_{1} q_{1}{ }^{2}+1 / 2 c_{3} r_{3}{ }^{2}+1 & { }_{2} r_{1} r_{4}{ }^{2} \\
& -2\left[F_{0}+F_{1} q_{1}{ }^{2}+F_{3} r_{3}{ }^{2}+F_{4} r_{4}{ }^{2}\right] \cos (\zeta / L) \\
& +2\left[G_{0}+G_{1} q_{1}{ }^{2}+G_{3} r_{3}{ }^{2}+G_{4} r_{4}{ }^{2}\right] \cos (2 \zeta / L)
\end{aligned}
$$

as a possible approximate potential energy function for the ammonia molecule. Equation (14) is, of course, a very incomplete potential function. Cubic and quartic terms could be added. However, unless the coefficients of the cubic and quartic terms are strongly dependent on the inversion coordinate $\zeta$, they will not make appreciable contributions to the inversion splitting. Since the purpose of this investigation is to examine the inversion splitting these extra terms will not be included.

Since $q_{1}, q_{3 x}, q_{3 y}, q_{4 x}$, and $q_{4 y}$ reduce to dimensionless normal coordinates near the equilibrium configurations, the corresponding normal frequencies can be found by evaluating the second derivalives of $V / h c$ at an equilibrium configuration. One finds

$$
\omega_{i}=c_{i}-4 F_{i} \cos \left(\zeta_{0} / L\right)+4 G_{i} \cos \left(2 \zeta_{0} / L\right) \quad i=1,3,4 .
$$

Apart from an additive constant, which can be neglected, the potential function of Eq. (14) can be rewritten as

$$
\begin{aligned}
V / h c= & 1 / 2 \omega_{1} q_{1}{ }^{2}+1 / 2 \omega_{3} r_{3}{ }^{2}+1 / 2 \omega_{4} r_{4}^{2} \\
& +\left[2 G_{0}{ }^{2}+F_{0}^{\prime 2} / 4 G_{0}{ }^{\prime}\right]-2 F_{0}^{\prime} \cos (\zeta / L)+2 G_{0}{ }^{\prime} \cos (2 \zeta / L) \\
& +\left[-2 F_{1}\left(\cos (\zeta / L)-\cos \left(\zeta_{0} / L\right)+2 G_{1}(\cos (2 \zeta / L)\right.\right. \\
& \left.\left.-\cos \left(2 \zeta_{0} / L\right)\right)\right]\left(q_{1}{ }^{2}-1 / 2\right) \\
& +\left[-2 F_{3}\left(\cos (\zeta / L)-\cos \left(\zeta_{0} / L\right)\right)+2 G_{3}(\cos (2 \zeta / L)\right. \\
& \left.\left.-\cos \left(2 \zeta_{0} / L\right)\right)\right]\left(r_{3}{ }^{2}-1\right) \\
& +\left[-2 F_{4}\left(\cos (\zeta / L)-\cos \left(\zeta_{0} / L\right)\right)+2 G_{4}(\cos (2 \zeta / L)\right. \\
& \left.\left.-\cos \left(2 \zeta_{0} / L\right)\right)\right]\left(r_{4}{ }^{2}-1\right)
\end{aligned}
$$


where

$$
\begin{aligned}
& F_{0}^{\prime}=F_{0}+1_{2} F_{1}+F_{3}+F_{4}, \\
& G_{0}^{\prime}=G_{0}+1_{2} G_{1}+G_{3}+G_{4} .
\end{aligned}
$$

It is in this form that the potential will be used in the subsequent calculations. It should be observed that this potential has minima at $\zeta= \pm \zeta_{0}$ and $q_{1}=r_{3}=$ $r_{4}=0$ where $\cos \left(\zeta_{0} / L\right)=F_{0} / 4 G_{0}$. These correspond to the two expulibrium configurations of the molecule.

Having arrived at expressions for the kinetic and potential energies, one "an set up the vibrational Hamiltonian for the molecule. Eequation (9), which is a rigorous expression for the kinetic energy, is somewhat more general than in needed. Examination of this equation reveals that the term involving

$$
\left[\sin \tau \mathbf{R}_{3}+\cos \tau \mathbf{R}_{4}\right]^{2}
$$

gives contributions to the inversion splitting of the order of $B_{n}-B_{n}{ }^{t}$, namely of the order of $0.005 \mathrm{~cm}^{-1}$ for states with $n_{2}=0$, and of the order of $0.2 \mathrm{~cm}^{-1}$ for states with $n_{2}=1$ (see Table $\mathrm{I}$ ). This term will be neglected in the treatment of the inversion-vibration splitting since this is of the order of $0.8 \mathrm{~cm}^{-1}$ in states with $n_{2}=0$ and $35 \mathrm{~cm}^{-1}$ in states with $n_{2}=1$. A further simplification arises from the assumption that $\sigma$ never differs very much from $\sigma_{n}$. Thus, the vihrational kinetic energy can be approximated by

$$
\begin{aligned}
& T_{\mathrm{vil}}=1,2 a_{0}{ }^{2}\left(\cosh ^{2} \sigma_{11}-\sin ^{2} \zeta\right)\left(\delta \dot{\sigma}^{2}+\ddot{\zeta}^{2}\right) \\
& +{ }^{2}{ }_{2}\left(\dot{Q}_{3 x}^{2}+\dot{Q}_{3_{y y}}^{2}+\dot{Q}_{4 x}^{2}+\dot{Q}_{+y}^{2}\right) .
\end{aligned}
$$

With the introduction of the dimensionless coordinates defined by E(y. (10) and a dimensionless inversion coordinate

$$
x=5 L \quad\left(x_{n}=\zeta_{11}: L\right),
$$

the quantum mechanical vibrational Hamiltonian becomes

$$
H_{\mathrm{vib}}=H_{\mathrm{vib}}^{(1)}+H_{\mathrm{vi} i}^{(1)},
$$

where

$$
\begin{aligned}
& H_{\mathrm{vib} /}^{(u)} / k c=\frac{\omega_{1}}{2}\left(-\frac{\partial^{2}}{\partial q_{1}^{2}}+q_{1}^{2}\right)+\frac{\omega_{3}}{2}\left(-\frac{\partial^{2}}{\partial q_{3 x}^{2}}-\frac{\partial^{2}}{\partial q_{3 !}^{2}}+r_{3}^{2}\right) \\
& +\frac{\omega_{4}}{2}\left(-\frac{\partial^{2}}{\partial q_{4 x}^{2}}-\frac{\partial^{2}}{\partial q_{4 y}^{2}}+r_{4}^{2}\right) \\
& +\left(-D \frac{\partial^{2}}{\partial x^{2}}+2 G_{11}^{\prime}+\frac{F_{0}^{\prime 2}}{4 G_{10}^{\prime}{ }^{\prime}}-2 F_{0 \prime}^{\prime} \cos x+2 G_{0 \prime}^{\prime} \cos 2 x^{\prime}\right)
\end{aligned}
$$


and

$$
\begin{aligned}
H_{\mathrm{vib}}^{(1)} / h c=h_{1}(x)\left(q_{1}{ }^{2}-1 / 2\right)+h_{3}(x)\left(r_{3}{ }^{2}-1\right)+h_{4}(x)\left(r_{4}{ }^{2}-1\right) & \\
& -D\left[f_{1}(x) \frac{\partial^{2}}{\partial x^{2}}+f_{2}(x) \frac{\partial}{\partial x}\right]-{ }_{2}^{\omega_{1}} f_{1}(x)\left(\frac{\partial^{2}}{\partial q_{1}^{2}}+\frac{1}{2}\right),
\end{aligned}
$$

where

$$
h_{i}(x)=-2 F_{i}\left(\cos x-\cos x_{0}\right)+2 C_{i}\left(\cos 2 x-\cos 2 x_{0}\right) \quad i=1,3,4
$$

and

$$
\begin{gathered}
D=h /\left[8 \pi^{2} c a_{0}^{2}\left(\cosh ^{2} \sigma_{0}-\sin ^{2} \zeta_{0}\right) L^{2}\right] \\
f_{1}(x)=\frac{\sin ^{2}(L x)-\sin ^{2} \zeta_{0}}{\cosh ^{2} \sigma_{0}-\sin ^{2}(L x)} \\
f_{2}(x)=\frac{2 L\left(\cosh ^{2} \sigma_{0}-\sin ^{2} \zeta_{0}\right) \sin (L x) \cos (L x)}{\left[\cosh ^{2} \sigma_{0}-\sin ^{2}(L x)\right]^{2}}
\end{gathered}
$$

The transcription to quantum mechanics has been made in such a way that the volume element in configuration space is simply $d q_{1} d x d q_{3 x} d q_{3 y} d q_{4 x} d q_{4 y}$. It will be noted that $H_{\mathrm{vib}}^{(0)}$ is completely separable in the various coordinates and that the inversion splitting, to this approximation, depends only upon $x . H_{\text {vib }}^{(1)}$ on the other hand, contains the interaction terms between the inversion and the other vibrational coordinates. In the calculations to follow, $H_{\mathrm{vih}}^{(1)}$ will be treated through perturbation methods. This appears justified since the change in energy levels (as opposed to the change in inversion splitting) caused by the interactions is small compared to the values of the levels themselves.

\section{VIBRATIONAL ENERGIES OF $\mathrm{NH}_{3}$ IN LOWEST APPROXIMATION}

Consider the Schrödinger equation for the unperturbed Hamiltonian

$$
H_{\mathrm{vib}}^{(0)} \Psi_{\mathrm{vib}}^{(0)}=E_{\mathrm{vib}}^{(0)} \Psi_{\mathrm{vib}}^{(0)}
$$

As just remarked, this equation is separable and gives

$$
\begin{aligned}
E_{v i b}^{(0)} / h c & =E_{n_{2}}+\omega_{1}\left(n_{1}+1,6\right)+\omega_{3}\left(n_{3}+1\right)+\omega_{4}\left(n_{4}+1\right), \\
\Psi_{\mathrm{vib}}^{(0)} & =\psi_{n_{2} \pm}(x) \psi_{n_{1}}\left(q_{1}\right) \psi_{n_{3} l_{3}}\left(q_{3 x}, q_{3 y}\right) \psi_{n_{4} l_{4}}\left(q_{4 x}, q_{4 y}\right),
\end{aligned}
$$

where $\psi_{n_{1}}$ is a one-dimensional harmonic oscillator wave function while $\psi_{n_{3} l_{3}}$ and $\psi_{n_{4} l_{4}}$ are two-dimensional isotropic harmonic oscillator wave functions. $E_{n_{2}} \pm$ and $\psi_{n_{2}} \pm$ are eigenvalues and eigenfunctions of the one-dimensional equation which describes the inversion.

$$
H_{2} \psi(x)=E \psi(x)
$$


where

$$
\begin{gathered}
H_{2}=-D\left(\partial^{2} / \partial x^{2}\right)+\left[2 G_{0}{ }^{\prime}+\left(F_{0}^{\prime 2} / 4 G_{0}{ }^{\prime}\right)\right]-2 F_{0}^{\prime} \cos x+2 G_{0}^{\prime} \cos 2 x \\
\text { for }-\pi \leqq x \leqq \pi, \\
H_{2}=-D\left(\partial^{2} / \partial x^{2}\right)+\left[2 G_{0}{ }^{\prime}+\left(F_{0}^{\prime 2} / 4 G_{x_{0}}{ }^{\prime}\right)\right]+2\left(F_{11}{ }^{\prime}+\left(x_{0}^{\prime}\right)\right. \\
\text { for } \pi<|x|<\pi 2 L .
\end{gathered}
$$

It does not appear possible to obtain exact solutions of equation (28) in terms of known functions. However, for $E \ll V(\pi)$ eigenvalues and eigenfunctions may be calculated numerically to any desired order of approximation. The proper procedure would be to obtain solutions in the interval $-\pi \leqq x \leqq+\pi$ and join them to the exponential solutions in the intervals $\pi<|x| \leqq \pi, 2 L$. However, for the levels of interest in the present investigation $\psi(x)$ becomes vanishingly small as $|x| \rightarrow \pi$ so that the solutions in the intervals $\pi<|x| \leqq \pi^{2} 2 L$ an be neglected entirely. Consequently, only solutions in the interval $-\pi \leqq$ $x \leqq+\pi$ need be considered.

Since the operator $H_{2}$ is unchanged when $x$ is replaced by $-x$, the eigenfunctions must be either even or odd functions of $x$. The form of $H_{2}$ suggests that $\psi(x)$ should be expanded in a Fourier series in the interval $-\pi \leqq x \leqq+\pi$. As a romputational procedure only the first $N$ terms of the Fourier' ('xpansions will bretained. Thus, the even functions become'

$$
\psi_{+}=\frac{a_{1)}}{\sqrt{2 \pi}}+\sum_{k=1}^{N-1} a_{k} \operatorname{Vos}_{\pi} \cos
$$

and the odd functions

$$
\psi_{-}=\sum_{k=1}^{N} \frac{b_{k}}{\sqrt{\pi}} \sin k x .
$$

Regarding these wave functions as $N$-parameter variational wave functions one obtains, by minimizing the usual variational integral with respect to the $a_{i}$ or $b_{i}$, the two matrix equations

$$
\begin{aligned}
& \sum_{k=0}^{N-1}\left[\left(H_{2}\right)_{j k}^{+}-E_{+} \delta_{j k}\right] a_{k}=0, \\
& \sum_{k=1}^{N}\left[\left(H_{2}\right)_{j k}^{-}-E_{-} \delta_{j k}\right] b_{k}=0,
\end{aligned}
$$

${ }^{2}$ For the $0^{+}$level, for example, the function $\psi(x)$ has a value of approximately 0.00005 at $x=\pi$ compared with a value of 0.94 at the maximum of the wave function. 
TABLE: II

Eigenvalues and Eigenvectors of $H_{2}{ }^{+}(12 \times 12$ Truncation $)$

\begin{tabular}{|c|c|c|c|c|c|}
\hline$n_{2}^{+}$ & $0^{+}$ & $1^{+}$ & $2^{+}$ & $3^{+}$ & $4^{+}$ \\
\hline $\begin{array}{l}E_{42^{+}} \\
\left(\mathrm{cm}^{-1}\right)\end{array}$ & 514.55 & 1451.71 & 2131.65 & 2899.49 & 3925.35 \\
\hline$a_{11}$ & 0.647154 & 0.125510 & 0.437270 & -0.131256 & 0.224109 \\
\hline$a_{1}$ & 0.362054 & 0.495878 & 0.307225 & 0.225158 & -0.131521 \\
\hline$a_{2}$ & -0.447480 & 0.603562 & 0.177672 & 0.357973 & -0.113213 \\
\hline$a_{3}$ & -0.467819 & -0.073639 & 0.575400 & -0.048483 & 0.371143 \\
\hline$a_{4}$ & -0.022430 & -0.531707 & 0.485977 & 0.269292 & 0.000916 \\
\hline$a_{5}$ & 0.160871 & -0.252730 & -0.102389 & 0.733106 & -0.014177 \\
\hline$a_{6}$ & 0.062313 & 0.097022 & -0.304725 & 0.353268 & 0.637748 \\
\hline$a_{7}$ & -0.020628 & 0.110248 & -0.089206 & -0.172564 & 0.578506 \\
\hline$a_{8}$ & -0.017838 & 0.009066 & 0.055148 & -0.188563 & -0.005545 \\
\hline$a_{9}$ & -0.000387 & -0.019376 & 0.034769 & -0.014796 & -0.192113 \\
\hline$a_{10}$ & 0.002506 & -0.005476 & -0.002021 & 0.034106 & -0.054597 \\
\hline$a_{11}$ & 0.000494 & 0.001508 & -0.005404 & 0.010167 & 0.020004 \\
\hline
\end{tabular}

where

$$
\begin{gathered}
\left(H_{2}\right)_{j k}^{ \pm}=\int_{-\pi}^{\pi} u_{j}^{ \pm} H_{2} u_{k}^{ \pm} d x, \\
u_{0}^{+}=1 / \sqrt{2 \pi}, \quad u_{k}^{+}=(1 / \sqrt{\pi}) \cos k x, \quad u_{k}^{-}=(1 / \sqrt{\pi}) \sin k x .
\end{gathered}
$$

Very good approximations to the low-lying eigenvalues and the corresponding eigenfunctions can be obtained by finding the eigenvalues and the eigenvectors of the two $\mathrm{N} \times \mathrm{N}$ matrices $\mathrm{H}_{2}{ }^{+}$and $\mathrm{H}_{2}{ }^{-}$, provided only that $\mathrm{N}$ is sufficiently large. The actual diagonalization was performed with the aid of the IBM 704 digital computer of The University of Michigan Computing Center.

By trial and error it was found that a value of $N$ equal to 12 was sufficiently large since an increase in $N$ beyond this value leads to insignificant changes in those eigenvalues and eigenfunctions which correspond to the presently observed levels in the ammonia spectrum. At least two cycles of computation are required to determine the constants $D, F_{0}{ }^{\prime}$, and $G_{0}{ }^{\prime}$. Although the decomposition of the vibrational Hamiltonian into $H_{\mathrm{vib}}^{(0)}$ and $H_{\mathrm{vib}}^{(1)}$ was made in a way that minimizes the contribution of $H_{\mathrm{vib}}^{(1)}$ to the levels $\left(0, n_{2}^{ \pm}, 0^{0}, 0^{0}\right)$, this contribution is not negligible. A tentative choice of $D, F_{0}{ }^{\prime}$, and $G_{0}{ }^{\prime}$ is made by neglecting $H_{\mathrm{vib}}^{(1)}$ altogether and choosing the three potential constants so that the best possible fit to the observed levels $\left(0, n_{2}^{ \pm}, 0^{0}, 0^{0}\right)$ is obtained. Using the resulting eigenfunctions, the approximate contribution due to $H_{\mathrm{vib}}^{(1)}$ is calculated. Then the values of $D, F_{0}{ }^{\prime}$, and $G_{0}{ }^{\prime}$ are adjusted to compensate for the contributions of $H_{v i b}^{(1)}$ to the levels $\left(0, n_{2}{ }^{ \pm}, 0^{0}, 0^{0}\right)$. The best values that have been found in this 
TABLE III

bigenvalues and Eigenvectors of $H_{2}(12 \times 12$ Truacation $)$

\begin{tabular}{|c|c|c|c|c|c|}
\hline$n \cdot 2^{-}$ & $0^{-}$ & $1^{-}$ & 2 & $3^{-}$ & 4 \\
\hline $\begin{array}{l}\sum_{n 2^{--}} \\
\left(\mathrm{cm}^{-1}\right)\end{array}$ & 515.49 & 1487.20 & 2401.77 & 3387.16 & 4482.53 \\
\hline$b_{1}$ & 0.771718 & -0.122245 & 0.368526 & -0.240582 & 0.254615 \\
\hline$b$. & 0.523913 & 0.481142 & $0.00+443$ & 0.298187 & $-0.26675 \times 3$ \\
\hline$b_{i:}$ & -0.148653 & 0.760754 & 0.107159 & 0.188733 & $0.086+27$ \\
\hline$b_{1}$ & -0.310924 & 0.174758 & 0.695148 & -0.110052 & $0.307 \times 27$ \\
\hline$b_{i}$ & -0.072999 & -0.313068 & 0.539354 & 0.448221 & $-0.151+17$ \\
\hline$b_{t}$ & 0.066 .367 & -0.206113 & -0.085132 & $0.71728 t$ & $0.21+791$ \\
\hline$b_{i}$ & 0.037308 & 0.021520 & $-0.25+207$ & 0.178893 & $0.728 t i l(0)$ \\
\hline$b$ & -0.003985 & 0.056222 & $-0.06+203$ & -0.205890 & 1).352643 \\
\hline$b_{4}$ & -0.007251 & 0.009731 & 0.040722 & -0.122294 & $-0.12974 !$ \\
\hline$b_{11}$ & -0.000800 & -0.007187 & 0.021746 & $0.0117+3$ & $-0.1+0729$ \\
\hline$t_{11}$ & 0.000775 & $-0.00263 i$ & -0.001966 & $0.02+031$ & $-0.00921 x$ \\
\hline$b_{1:}$ & 0.000206 & 0.000383 & -0.003106 & 0.003291 & 0.020130 \\
\hline
\end{tabular}

TABLE IV

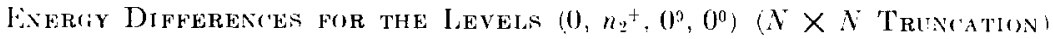

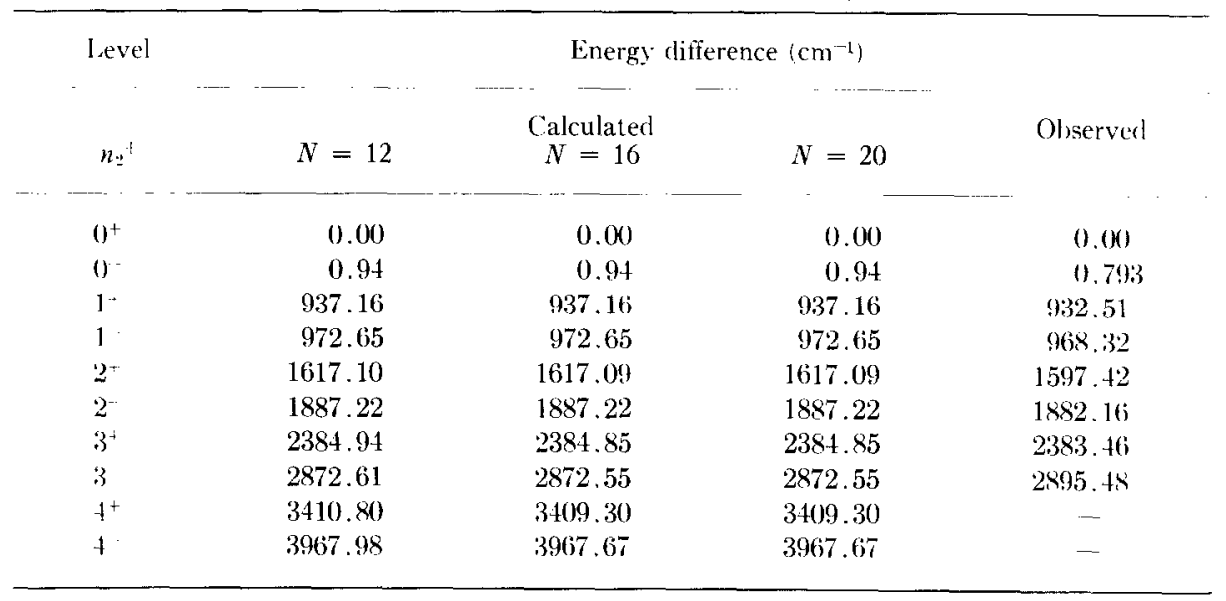

way are $I)=64.93 \mathrm{~cm}^{-1}, F_{0}^{\prime}=1894.61 \mathrm{~cm}^{1}$, and $\left(x_{0}^{\prime}=1288.68 \mathrm{~cm}^{-1}\right.$. Eigenvalues and eigenvectors of the matrices $\mathrm{H}_{2}{ }^{+}$and $\mathrm{H}_{2}{ }^{-}$have been computed using these values for the potential constants. The results, for $N$ equal to 12, are presented in Tables II and III. The calculated energy differences $E_{n=}^{ \pm}-E_{41}^{+}$for $N$ equal to 12,16 , and 20 , are given in Table IV along with the observed differences for the levels $\left(0, n_{2}{ }^{ \pm}, 0^{0}, 0^{\prime \prime}\right)$. However, comparison between theory 
and experiment should be made only after the contributions of $H_{\mathrm{vib}}^{(1)}$ have been computed and incorporated in the calculated values.

\section{INVERSION-VIBRATION INTERACTIONS}

Having obtained zeroth order energies and wave functions it is now possible to compute the contributions due to the perturbation $H_{\mathrm{vib}}^{(1)}$. This calculation is done in two steps. First, perturbation theory is used to express the energy corrections as functions of the interaction constants $F_{i}$ and $G_{i}(i=1,3,4)$. The second step is to choose numerical values of the interaction constants in such a way that a good overall description of the vibration-inversion splitting is obtained.

In the matrix elements of $H_{\mathrm{vib}}^{(1)}$ the integrals over the harmonic oscillator wave functions are well known. The integrals over the double minimum wavefunctions must, however, be evaluated numerically. The matrix of $H_{\mathrm{vib}}^{(1)}$ is diagonal in the quantum numbers $l_{3}$ and $l_{4}$ and moreover has no matrix elements connecting + states with - states. Since there are no matrix elements connecting states of the same unperturbed energy, nondegenerate first- and second-order perturbation theory can be used to calculate the energy corrections.

In order to achieve the desired goal of expressing the energy corrections as functions of the interaction constants alone it is necessary to know the numerical values of the normal frequencies $\omega_{1}, \omega_{3}$, and $\omega_{4}$. Furthermore, in order to do the numerical integrations over the inversion coordinate $x$ one must assign numerical values to the five parameters $a_{0}, \zeta_{0}, \sigma_{0}, L$, and $x_{0}$. The most recent estimates of the normal frequencies and equilibrium moments of inertia of $\mathrm{NH}_{3}$ and $\mathrm{ND}_{3}$ have been made by Benedict and Plyler (20) and their values for $\omega_{1}, \omega_{3}$, and $\omega_{4}$ were used in the present calculations. The equilibrium dimensions $u_{1}^{0}$ and $u_{2}{ }^{0}$ were obtained from their estimates of the equilibrium moments of inertia. The constant $a_{0}$ can be calculated once the quadratic force constants $a, b$, and $c$ for the symmetric vibrations are known. These are calculated from the normal frequencies $\left(\omega_{1}\right)_{\mathrm{NH}_{3}},\left(\omega_{1}\right)_{\mathrm{ND}_{3}}$, and $\left(\omega_{2}\right)_{\mathrm{NH}_{3}}$. For the first two of these frequencies the values given by Benedict and Plyler were used. The normal frequency $\left(\omega_{2}\right)_{\mathrm{NH}_{3}}$ can be calculated from the potential energy function given by Eqs. (14) or (16). One finds

$$
\left(\omega_{2}\right)^{2}=16 D G_{0}\left[1-\left(F_{0} / 4 G_{0}\right)^{2}\right] .
$$

The constants $F_{0}$ and $G_{0}$ are as yet unknown. However, from the known constants $F_{0}^{\prime}=F_{0} \mid\left(\frac{1}{2}\right) F_{1}+F_{3}+F_{4}$ and $G_{0}^{\prime}=G_{0}+(1 / 2) G_{1}+G_{3}+G_{4}$ an approximate value for $\left(\omega_{2}\right)_{\mathrm{NH}_{3}}$ can be found, assuming the $F_{1}, F_{3}$, and $F_{4}$ are much smaller than $F_{0}$, and that $G_{1}, G_{3}$, and $G_{4}$ are much smaller than $G_{0}$. In the final cycle of the calculation an improved value of $\left(\omega_{2}\right)_{\mathrm{NH}_{3}}$ derived from the constants $F_{0}$ and $G_{0}$ will be used. The energies, including contributions due to $H_{\mathrm{vib}}^{(1)}$, are given by 


$$
E_{\mathrm{vib}}=E_{\mathrm{vib}}^{(1)}+E_{\mathrm{vib}}^{(1)},
$$

where $E_{\mathrm{vib}}^{(1)}$ is the zeroth order energy given by Fq. (26) and $E_{\mathrm{vib}}^{(1)}$ is a function of the six interaction constants $F_{i}$ and $G_{i}(i=1,3,4)$.

In order to describe the method by which the interaction constants are determined it is eonvenient to introduce the notation

$$
\begin{aligned}
\Delta E\left(n_{1} n_{2} n_{3}^{l_{3}} n_{4}^{l^{4}}\right) & =E\left(n_{1} n_{2}{ }^{-} n_{3}^{l_{3}} n_{4}^{l_{4}}\right)-E\left(n_{1} n_{2}{ }^{+} n_{3}^{l_{3}} n_{4}^{l_{4}}\right), \\
\bar{E}\left(n_{1} n_{2} n_{3}^{l_{3}} n_{4}^{l_{4}}\right) & =1_{2}\left[E\left(n_{1} n_{2}{ }^{-} n_{3}^{l_{3}} n_{4}^{l_{4}}\right)+E\left(n_{1} n_{2}{ }^{+} n_{3}^{l_{3}} n_{4}^{l_{4}}\right)\right] .
\end{aligned}
$$

Thus, $\Delta E$ is the splitting of the pair of levels $\left(n_{1}, n_{2}{ }^{ \pm}, n_{3}^{l_{3}}, n_{4}^{l_{4}}\right)$ and $\bar{E}$ is their mean position. For simplicity, the splittings $\Delta E\left(0, n_{2}, 0^{n}, 0^{0}\right)$ will be denoted by $\Delta_{n: 3}$. The constants $F_{3}$ and $G_{3}$ can be determined from the observed shift in splitting $\Delta E\left(\begin{array}{llllll}0 & 0 & 1^{1} & 0^{0}\end{array}\right)-\Delta_{0}$ and the observed anharmonicity $\bar{E}\left(\begin{array}{llll}0 & 1 & 1^{1} & 0^{\prime \prime}\end{array}\right)-$

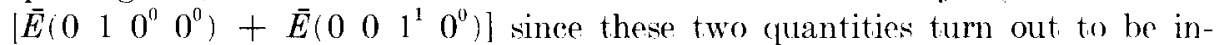
dependent of $F_{1}, G_{1}, F_{4}$, and $r_{4}$. The observed numbers yicld

$$
F_{3}=-175.0 \mathrm{~cm} \mathrm{~m}^{-1}, \quad G_{r 3}^{r}=14.0 \mathrm{~cm}^{-1} .
$$

Similarly, the shift in splitting $\Delta E\left(\begin{array}{llll}0 & 0 & 0^{0} & 1^{1}\end{array}\right)-\Delta_{0}$ and the anharmonicity

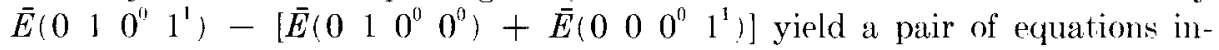
volving $F_{4}$ and $G_{4}$ alone which give

$$
F_{4}=29.7 \mathrm{~cm}^{-1}, \quad i_{4}=-13.8 \mathrm{~cm}^{-1} .
$$

The question naturally arises why the two observed shifts in splitting

$$
\Delta E\left(\begin{array}{llll}
0 & 0 & 1^{1} & \left.0^{a}\right)-\Delta_{0}
\end{array} \text { and } \Delta E\left(\begin{array}{llllll}
0 & 1 & 1^{1} & 0^{0}
\end{array}\right)-\Delta_{1}\right.
$$

wore not used to determine $F_{3}$ and $r_{3}$. Unfortunately one finds that the two equations for $F_{3}$ and $G_{3}$ obtained in this way are almost constant multiples of each other and consequently, with an interaction of the form postulated above, the two observed splittings with $n_{3}=1$ are not, effectively, independent pieces of information. Two pieces of information of a distinctly different nature are needed and the observed anharmonicity and one of the observed splittings: sutisfy this requirement.

The determination of $F_{1}$ and $G_{1}$ is subject to a much greater uncertainty than i.s the rase for $F_{3}, G_{3}, F_{4}$, and $r_{4}$. Three pairs of levels,

$$
\left(1,0^{ \pm}, 0^{0}, 0^{\prime \prime}\right), \quad\left(1,1^{ \pm}, 0^{0}, 0^{0}\right), \quad \text { and } \quad\left(1,0^{ \pm}, 0^{0}, 1^{1}\right)
$$

involving the excitation of the $q_{1}$ mode have been observed in the spectrum of $\mathrm{NH}_{3}$. Infortunately,

$$
E_{\mathrm{vil}}\left(1,0^{ \pm}, 0^{\prime \prime}, 0^{\prime \prime}\right) \quad \text { and } \quad E_{\text {vill }}^{\prime}\left(0,0^{ \pm}, 0^{0}, 2^{\prime \prime}\right)
$$

differ only by about $120 \mathrm{~cm}^{-1}$, and these levels may be in Fermi resonance with each other through a cubie potential term of the form $K_{144} q_{1} r_{4}{ }^{2}$ which has been 
neglected in the potential function used in this investigation. Similarly, the levels $\left(1,1^{ \pm}, 0^{0}, 0^{0}\right)$ may be in resonance with $\left(0,1^{ \pm}, 0^{0}, 2^{0}\right)$ and $\left(1,0^{ \pm}, 0^{0}, 1^{1}\right)$ may be in resonance with $\left(0,0^{ \pm}, 0^{0}, 3^{1}\right)$. (The levels $\left(0,0^{ \pm}, 0^{0}, 3^{1}\right)$ and $\left(0,1^{ \pm}, 0^{0}, 2^{0}\right)$ have not yet been ubserved in the ammonia spectrum.) In general, one would expect the Fermi resonance to have some effect on the splittings of the levels involved. Similarly, the observed anharmonicity

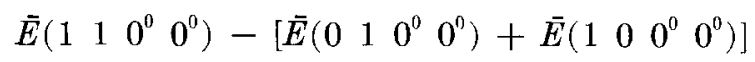

can be expected to involve a contribution due to the Fermi resonance. No matter whether the observed numbers or estimated values of the unperturbed numbers, corrected for Fermi resonance, are used, one has the problem of choosing $F_{1}$ and $G_{1}$ such that

$$
\begin{aligned}
\Delta E\left(\begin{array}{llll}
1 & 0 & 0^{0} & 0^{0}
\end{array}\right)>\Delta_{0}, \\
\Delta E\left(\begin{array}{llll}
1 & 1 & 0^{0} & 0^{0}
\end{array}\right)<\Delta_{1} .
\end{aligned}
$$

It appears that no values of $F_{1}$ and $G_{1}$ exist, unless third and higher order perturbations give significant contributions, which will satisfy these inequalities. One can fit the splitting of the levels $\left(10^{ \pm}, 0^{\circ}, 0^{\circ}\right)$, in which case the predicted splitting of the levels $\left(1,1^{ \pm}, 0^{0}, 0^{0}\right)$ is much too large or one can fit the splitting of the levels $\left(1,1^{ \pm}, 0^{0}, 0^{0}\right)$, in which case the calculated splitting of the levels $\left(1,0^{ \pm}, 0^{0}, 0^{0}\right)$ will be too small. In order to obtain a rough estimate of the values of $F_{1}$ and $G_{1}$ the second alternative was chosen. Although the choice is quite arbitrary it might be hoped that the lermi resonance has a smaller percentage influence on the splitting of the levels $\left(1,1^{ \pm}, 0^{0}, 0^{\circ}\right)$ than on the splitting of the levels $\left(1,0^{ \pm}, 0^{0}, 0^{0}\right)$. The values of $F_{1}$ and $G_{1}$ obtained in this way are

$$
F_{1}=-144.0 \mathrm{~cm}^{-1}, \quad G_{1}=25.6 \mathrm{~cm}^{-1} .
$$

It should be understood that the numbers for $F_{1}$ and $G_{1}$ may be badly in error. In fact, the dilemma cited above casts doubt upon the validity of the interaction function between $q_{1}$ and $x$ which has been postulated.

Since $F_{0}^{\prime}$ and $G_{0}{ }^{\prime}$ are known, the values of the interaction constants can be used, with the aid of Eq. (17), to calculate $F_{0}$ and $G_{0}$. Equation (31) then yields an improved value for the normal frequency $\omega_{2}$ for $\mathrm{NH}_{3}$, which in turn can be used to obtain corrected estimates of the force constants $a, b$, and $c$. Improved estimates of the parameters $a_{0}, \zeta_{0}, \sigma_{0}, L$, and $x_{0}$ then can be made. The best values obtained for these parameters are presented in Table V. As a final step, the corrections to the energy levels due to $H_{\mathrm{vib}}^{(1)}$ have been recalculated.

It should be noticed that the magnitudes of the interaction constants $F_{i}$ and $G_{i}$ are sufficiently small to justify the use of perturbation theory. That this would be so was not obvious at the beginning of the calculation. Another point of interest is that $H_{\mathrm{vib}}^{(1)}$ contributes a correction to the levels $\left(0, n_{2}^{ \pm}, 0^{0}, 0^{9}\right)$. 
TABIL $V$

Parameters fOR $\mathrm{NH}_{3}$

\begin{tabular}{|c|c|c|c|}
\hline $\begin{array}{l}\omega_{1} \\
\omega_{i:} \\
\omega_{1}\end{array}$ & $\begin{array}{l}=3516.98 \mathrm{~cm}^{-1} \\
=3590.51 \mathrm{~cm}^{-1} \\
=1689.11 \mathrm{~cm}^{-1}\end{array}$ & $\begin{array}{l}(3 m)^{1 / 2} u_{1} \\
(\mu)^{1 / 2} u_{2}{ }^{0}\end{array}$ & $\begin{array}{l}=2.101 \times 10^{-20} \mathrm{~cm} \times \mathrm{g}^{1 / 2} \\
=0.7757 \times 10^{-20} \mathrm{~cm} \times \mathrm{g}^{1 / 2}\end{array}$ \\
\hline$F_{u i}^{\prime}$ & $=1894.61 \mathrm{~cm}^{-1}$ & $G_{4}$ & $=-13.8 \mathrm{~cm}^{-1}$ \\
\hline$G_{10}{ }^{\prime}$ & $=1288.68 \mathrm{~cm}^{-1}$ & $x_{11}$ & $=1.1441$ \\
\hline D) & $=64.93 \mathrm{~cm}^{-1}$ & $\sinh \sigma_{0}$ & $=1.1133$ \\
\hline$F_{11}$ & $=2111.91 \mathrm{~cm}^{-1}$ & $\sin \zeta_{0}$ & $=0.2648$ \\
\hline$G_{11}$ & $=1275.68 \mathrm{~cm}^{\cdots 1}$ & $L$ & $=0.2342$ \\
\hline$F_{1}$ & $=-144.0 \mathrm{~cm}^{-1}$ & $a_{0}$ & $=1.9574 \times 10^{-20} \mathrm{~cm} \times \mathrm{g}^{1 / 2}$ \\
\hline$G_{1}$ & $=25.6 \mathrm{~cm}^{-1}$ & $\omega:$ & $=1047.98 \mathrm{~cm}^{-1}$ \\
\hline$F_{: 3}$ & $=-175.0 \mathrm{~cm}-1$ & $a$ & $=2.2712 \times 10^{6} \mathrm{dyne} / \mathrm{cm}$ \\
\hline$G_{?}$ & $=14.0 \mathrm{~cm}^{-1}$ & $b$ & $=7.0770 \times 10^{5} \mathrm{dyne} / \mathrm{cm}$ \\
\hline$F_{1}$ & $=29.7 \mathrm{~cm}^{-1}$ & $c$ & $=2.0624 \times 10^{5} \mathrm{dyne} / \mathrm{cm}$ \\
\hline
\end{tabular}

Since the major contributions to the energy corrections of the lower states come from matrix elements of $H_{\mathrm{vib}}^{(1)}$ with $\Delta n_{2}=0, \pm 1$, and since it is estimated that contributions of matrix elements with $n_{2} \geqq 4$ are less important for these states than the effects of third or higher order perburbation terms, only matrix elements $\left(n_{2}\left|H_{\mathrm{vib}}^{(1)}\right| n_{2}^{\prime}\right)$ with $n_{2}, n_{2}^{\prime}<4$ have been retained for simplicity, and the corrections for states, $n_{2}=3$, have not been included. The corrected energies for the levels $\left(0, n_{2}^{ \pm}, 0^{n}, 0^{0}\right)$ for $n_{2}=0,1$, and 2 are given in Table VI. In this table two sets of ealculated energy values are given. For the first of these the simple form of the inversion potential, assumed up to now in this investigation, has been used for the zeroth order calculation. The corrected energies should be compared with the unperturbed energies given in Table IV. It is sech that the inclusion of the corrections due to $H_{\mathrm{vib}}^{(1)}$ improves the agreement between calculated and observed energies. However, the ground state splitting remains too large even after the correction has been applied. With the simple form of the inversion potential used so far it does not seem possible to decrease the ground state splitting and still retain a very good overall description of the remaining $\left(0, n_{2}{ }^{ \pm}, 0^{0}, 0^{0}\right)$ levels. However, only a very slight modification of the inversion potential function is needed to give an excellent fit between the observed and predicted levels. In one possible generalization the simple form of the potential function used so far can be regarded as consisting of the leading and dominant terms in a Fourier expansion of the true potential function. Better approximations might then be obtained by including $\cos 3 x, \cos 4 x$, or higher terms. It is found that it is possible to fit both the observed $n_{2}=0$ and $n_{2}=1$ levels with the inclusion of a small negative $\cos 4 x$ term, that is with the following modified form of the effective inversion potential function

$$
r_{1 \prime}^{\prime}\left(x^{\prime}\right)=2 x_{0}^{\prime}+F_{11}^{\prime 2} 4 x_{0}^{\prime}-2 F_{0}^{\prime} \cos x+2 C_{0 \prime}^{\prime} \cos 2 x-2 \mu_{0} \cos 4 x \text {. }
$$


TABLE VI

Corrected Energy Levelas $E_{\text {vib }}\left(0, n_{2^{ \pm}}, 0^{0}, 0^{0}\right)$

\begin{tabular}{|c|c|c|c|}
\hline Level & $\begin{array}{l}\text { Calculated }\left(\mathrm{cm}^{-1}\right) \\
\text { Simple form of } \\
\text { inversion potential }\end{array}$ & Observed $\left(\mathrm{cm}^{-1}\right)$ & $\begin{array}{r}\text { Calculated }\left(\mathrm{cm}^{-1}\right) \\
\text { Modified form of } \\
\text { inversion potential }\end{array}$ \\
\hline 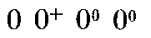 & 0.00 & 0.00 & 0.00 \\
\hline $\begin{array}{llll}0 & 0^{-} & 0^{0} & 0^{0}\end{array}$ & 0.88 & 0.793 & 0.79 \\
\hline $01^{+} 0^{0} 0^{0}$ & 933.20 & 932.51 & 932.52 \\
\hline $01^{-} 0^{0} 0^{0}$ & 968.29 & 968.32 & 968.33 \\
\hline $02^{+} 0^{0} 0^{0}$ & 1606.70 & 1597.42 & 1588.24 \\
\hline 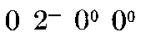 & 1877.78 & 1882.16 & 1868.25 \\
\hline Splitting & $\begin{array}{c}\text { Calculated }\left(\mathrm{cm}^{-1}\right) \\
\text { Simple form of } \\
\text { inversion potential }\end{array}$ & Observed $\left(\mathrm{cm}^{-1}\right)$ & $\begin{array}{l}\text { Calculated }\left(\mathrm{cm}^{-1}\right) \\
\text { Modified form of } \\
\text { inversion potential }\end{array}$ \\
\hline$\Delta_{0}$ & 0.88 & 0.793 & 0.79 \\
\hline$\Delta_{1}$ & 35.09 & 35.81 & 35.81 \\
\hline$\Delta_{2}$ & 271.08 & 284.56 & 280.01 \\
\hline
\end{tabular}

The calculated energy values given in the last column of Table VI (which also include the corrections due to $H_{\mathrm{vib}}^{(1)}$ ) were obtained with the following values of the parmeters: $\mu_{0}=21.80 \mathrm{~cm}^{-1}, F_{0}^{\prime}=1,888.81 \mathrm{~cm}^{-1}, G_{0}^{\prime}=1,287.65 \mathrm{~cm}^{-1}$, and $D=64.71 \mathrm{~cm}^{-1}$. Comparison with Table $\mathrm{V}$ shows that the inclusion of the small cos $4 x$ term necessitates a slight adjustment of the dominant potential parameters. With the addition of still further small correction terms it would be possible to improve the fit of the higher inversion states. No such refinements however will be made sine the main purpose of the present investigation is to account for the dependence of the inversion splittings on the vibrational and rotational quantum numbers. For all subsequent calculations therefore only the simple form of the effective inversion potential (without the $\cos 4 x$ term) will be used.

The calculated inversion splitting, as a function of the vibrational quantum numbers, for levels with $n_{2}$ equal to 0 or 1 is given by

$$
\begin{aligned}
\Delta E\left(n_{1} 0 n_{3}^{l_{3}} n_{4}^{l_{4}}\right)=\Delta_{0}- & 0.56 n_{1}-0.73 n_{3}+0.22 n_{4} \\
& +0.27 n_{1}{ }^{2}+0.28 n_{3}{ }^{2}+0.03 n_{4}{ }^{2}-0.17 n_{3} n_{4} \\
& +0.78 n_{1} n_{3}-0.18 n_{1} n_{4}-0.0003 l_{3}{ }^{2}-0.0007 l_{4}{ }^{2}
\end{aligned}
$$

and

$$
\begin{aligned}
\Delta E\left(n_{1} 1 n_{3}^{l_{3}} n_{4}^{l_{4}}\right)=\Delta_{1}- & 16.44 n_{1}-22.63 n_{3}+6.75 n_{4} \\
+ & 5.95 n_{1}{ }^{2}+6.21 n_{3}{ }^{2}+0.54 n_{4}{ }^{2}-3.69 n_{3} n_{4} \\
+ & 12.16 n_{1} n_{3}-3.60 n_{1} n_{1}-0.0057_{3}{ }^{2}-0.002 l_{4}{ }^{2}
\end{aligned}
$$


An inspertion of these equations suggests that, regarded as power series derelopments in the quantum numbers, they may not be rapidly convergent. (ubic and quartic terms would have been obtained through third and higher order perturbation corrections but this lies outside the seope of the present paper.

The calculated splittings for levels with $n_{1}=0$ are compared with the observed splittings in Table VII. These numbers were obtained from F(1s. (32) and (33) by setting $\Delta_{0}$ and $\Delta_{1}$ equal to their observed values 0.793 and 35.81 $\left(\cdot \mathrm{n}^{-1}\right.$, respectively. The numbers in Table VII are sensitive functions of $F_{33}$, $G_{3}, F_{4}$, and $G_{4}$ but are essentially independent of $F_{1}$ and $G_{1}$. It is seen that the ralculated and observed results agree reasonably well with the exception of the splitting of the levels $\left(0,0^{ \pm}, 0^{\prime \prime}, 2^{n}\right)$. It was pointed out above that this pair of levels may be in lermi resonance with the pair $\left(1,0^{ \pm}, 0^{n}, 0^{0}\right)$ and conscequently a good agreement is perhaps not to be expected. Apart from the exception just mentioned, eleven data have been accounted for with four interaretion ronstants. No doubt a still better agreement could be obtained by introduring further parameters in the potential function and by arying ont the perturbation calculation to higher orders. The fact that the calculated mumbers do not reproduce exactly the data used to determino the interaction ensitants

TABLE VII

Inversion-Vibration Interactiona: Degengrate Vibrationi

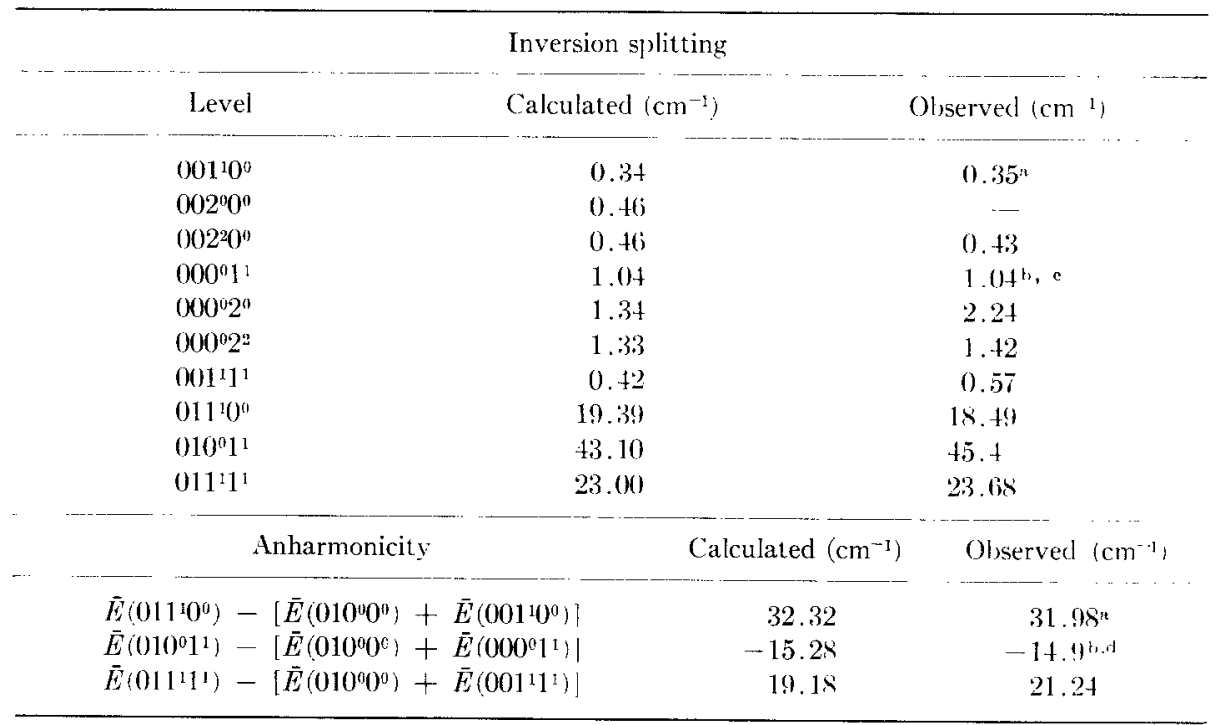

" Used to fil $F_{3}$ and $G_{3}$.

b. Used to fit $F_{4}$ and $G_{4}$.

"A more recent value is $1.01 \mathrm{~cm}^{-1}$-see Ref. 5 .

"In view of Ref. $5,-16.88\left(\mathrm{~m}^{-1}\right.$ is probably hetter. 
is due primarily to the fact that these constants were obtained using approximate values of the parameters $a_{0}, \zeta_{0}, \sigma_{0}, L$, and $x_{0}$, whereas the numbers given in Table VII were calculated using improved values for these parameters. Table VIII summarizes the results for the splittings of states in which the symmetric $q_{1}$ vibration has been excited. The agreement is seen to be relatively poor. The anharmonicity used to determine $F_{1}$ and $G_{1}$ should be the one corrected for Fermi resonance, namely, $20.18 \mathrm{~cm}^{-1}+C_{\mathrm{FR}}\left(100^{0} 0^{0}\right)-C_{\mathrm{FR}}\left(110^{0} 0^{\circ}\right)$, where $C_{\mathrm{FR}}$ is the correction due to Fermi resonance. Benedict et al. (4) have estimated that $C_{\mathbf{F R}}\left(100^{0} 0^{0}\right)$ is $13.5 \mathrm{~cm}^{-1}$. Since the levels $\left(0,1^{ \pm}, 0^{0}, 2^{0}\right)$ have not been observed it is difficult to make a reasonable estimate of $C_{\mathbf{F R}}\left(110^{0} 0^{\circ}\right)$. For the purpose of determining $F_{1}$ and $G_{1}$ a somewhat arbitrary estimate of $10 \mathrm{~cm}^{-1}$ was made for the overall contribution of the Fermi resonance to the anharmonicity.

It might be thought that the discrepancy between the romputed and observed splitting for the pair of levels $\left(1,0^{ \pm}, 0^{0}, 0^{0}\right)$ can be attributed entirely to a Fermi resonance with the pair $\left(0,0^{ \pm}, 0^{0}, 2^{0}\right)$. The effects of Fermi resonance, however, cannot be the only source of the error since it can be shown (11) that the sum of the splittings for the states $\left(1,0^{ \pm}, 0^{0}, 0^{0}\right)$ and $\left(0,0^{ \pm}, 0^{0}, 2^{0}\right)$ is unchanged by the resonance whereas the predicted splittings (Tables VII and VIII) are too small in both cases. The trouble may lie in the simple form assumed for the interaction between $q_{1}$ and $x$. Further experimental information about states such as $\left(2,0^{ \pm}, 0^{0}, 0^{0}\right)$ or $\left(1,2^{ \pm}, 0^{0}, 0^{0}\right)$ might help to clear up this difficulty.

\section{VIBRATIONAL ENERGIES OF $\mathrm{ND}_{3}$ IN LOWEST APPROXIMATION}

As a test of the validity of the inversion potential chosen for the ammonia molecule it will be interesting to calculate the set of vibrational levels $\left(0, n_{2}{ }^{ \pm}\right.$, $0^{0}, 0^{0}$ ) for $\mathrm{ND}_{3}$. As is well known, the potential functions for two isotopic species of a molecule are identical to a very high order of approximation. This

\section{TABLE VIII}

INVERSION-VIBRATION INTERACTIONS: LEVELS WITH $n_{1}=1$

\begin{tabular}{ccc}
\hline & \multicolumn{2}{c}{ Inversion splitting } \\
\hline Level & Calculated $\left(\mathrm{cm}^{-1}\right)$ & Observed $\left(\mathrm{cm}^{-1}\right)$ \\
\hline $100^{0} 0^{0}$ & 0.51 & 0.99 \\
$101^{1} 0^{0}$ & 0.81 & - \\
$100^{\circ} 1^{1}$ & 0.58 & 0.86 \\
$110^{\circ} 0^{0}$ & 25.32 & 25.55 \\
\hline Anharmonicity & Calculated $\left(\mathrm{cm}^{-1}\right)$ & Observed $\left(\mathrm{cm}^{-1}\right)$ \\
\hline $\bar{E}\left(110^{\circ} 0^{\circ}\right)-\left[\bar{E}\left(010^{\circ} 0^{\circ}\right)+\bar{E}\left(100^{\circ} 0^{\circ}\right)\right]$ & 30.63 & $30.18 \mathrm{~cm}^{\mathrm{a}}$ \\
\hline
\end{tabular}

a Including a somewhat arbitrary correction of $10 \mathrm{~cm}^{-1}$ for Firmi resonance. 
identity of potential function imposes the following relations between the constants of $\mathrm{Eq}$. (14) and those of the corresponding formula for $\mathrm{ND}_{3}$.

$$
\begin{aligned}
& \left(F_{0}\right)_{\mathrm{ND}_{3}}=\left(F_{0}\right)_{\mathrm{NH}_{3}}, \quad\left(G_{0}\right)_{\mathrm{ND}_{3}}=\left(G_{0}\right)_{\mathrm{NH}_{3}},
\end{aligned}
$$

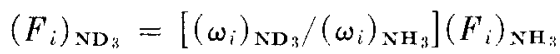

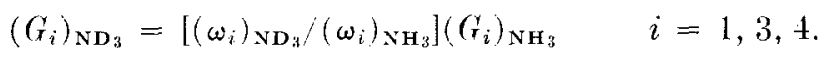

$$
\begin{aligned}
& \left(c_{i}\right)_{\mathrm{ND}_{3}}=\left[\left(\omega_{i}\right)_{\mathrm{ND}_{3}} /\left(\omega_{i}\right)_{\mathrm{NH}_{3}}\right]\left(c_{i}\right)_{\mathrm{NH}_{3}}
\end{aligned}
$$

These relations follow at once from the fact that the ratio $(\zeta / L)$ is a mass independent, dimensionless quantity while the coordinates $q_{1}, r_{3}$, and $r_{4}$ are dimensionless small oscillation coordinates related to the w's in the usual way. The transformation of the quantity $D$ which occurs in the kinetic energy part of the Hamiltonian is accomplished through Eq. (31) and is

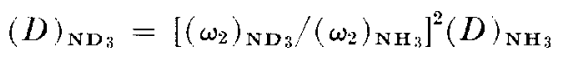

The normal frequency $\left(\omega_{2}\right)_{\mathrm{ND}_{3}}$ can be obtained through the aid of the observed $\omega_{1}$ by applying the product rule

$$
\frac{\left(\omega_{1}\right)_{\mathrm{ND}_{3}}\left(\omega_{2}\right)_{\mathrm{ND}_{3}}}{\left(\omega_{1}\right)_{\mathrm{NH}_{3}}\left(\omega_{2}\right)_{\mathrm{NH}_{3}}}=\frac{m_{H}}{m_{D}}\left[\frac{M+3 m_{D}}{M+3 m_{H}}\right]^{1 / 2}
$$

where $I I$ is the nitrogen mass, $m_{\mathrm{H}}$ the hydrogen mass, and $m_{\mathrm{D}}$ the deuterium mass.

The values of the constants for $\mathrm{ND}_{3}$, calculated from $\mathrm{Eq}$. (34), are given in Table IX along with the constants $F_{0}^{\prime}$ and $G_{0}^{\prime}$ appearing in the effective onedimensional potential. Using these values of the constants the eigenvalues of the one-dimensional double minimum problem, Eq. (28), (simple form of the inversion potential), have been found for $\mathrm{ND}_{3}$ according to the method described in Section IV. In this case, the $0^{+}$level lies $394.93 \mathrm{~cm}^{-1}$ above the minima of the effective one-dimensional potential. The energy differences $E_{n, 2}^{+}-E_{n}^{+}$ are given in Table $\mathrm{X}$.

\begin{tabular}{|c|c|c|}
\hline & $\begin{array}{l}\omega_{1}=2496.96 \\
\omega_{3}=2642.18 \\
\omega_{4}=1226.32\end{array}$ & \\
\hline $\begin{array}{l}\omega_{2}=801.01 \\
D=37.93 \\
F_{0}=2111.91 \\
G_{0}=1275.68 \\
F_{1}=-102.2 \\
G_{1}=18.2\end{array}$ & & $\begin{aligned} F_{3} & =-128.8 \\
G_{3} & =10.3 \\
F_{4} & =21.6 \\
G_{4} & =-10.0 \\
F_{0}^{\prime} & =1953.61 \\
G_{0}^{\prime} & =1285.08\end{aligned}$ \\
\hline
\end{tabular}

TABLE IX

Constants for $\mathrm{ND}_{3}\left(\mathrm{~cm}^{-1}\right)$ 
TABLE $\times$

Energy Differences for the Levels $\left(0, n_{9^{ \pm}}, 0^{0}, 0^{0}\right)$ of $N^{\prime}(N \times N$

Truncation)

\begin{tabular}{|c|c|c|c|c|c|c|}
\hline \multirow{3}{*}{$\begin{array}{l}\text { Level } \\
n_{2} \pm\end{array}$} & \multicolumn{6}{|c|}{ Energy differences $\left(\mathrm{cm}^{-1}\right)$} \\
\hline & \multicolumn{3}{|c|}{ Calculated simple form of inversion potential } & \multirow{2}{*}{$\begin{array}{l}\text { Calculated modi- } \\
\text { fed form of } \\
\text { inversion polential } \\
N=16\end{array}$} & \multirow{2}{*}{$\begin{array}{c}\text { Estimated } \\
\text { perturbation } \\
\text { correction }\end{array}$} & \multirow[t]{2}{*}{ Observed } \\
\hline & $N=12$ & $N=16$ & $N=20$ & & & \\
\hline $0^{+}$ & 0.00 & 0.00 & 0.00 & 0.00 & & 0.00 \\
\hline $0^{-}$ & 0.08 & 0.08 & 0.08 & 0.07 & -0.02 & 0.053 \\
\hline $1^{+}$ & 746.90 & 746.74 & 746.74 & 748.18 & -2.0 & 745.7 \\
\hline $1^{\sim}$ & 751.20 & 751.18 & 751.18 & 752.61 & -2.2 & 749.4 \\
\hline $2^{+}$ & 1359.71 & 1359.45 & 1359.45 & 1347.44 & -5 & 1359 \\
\hline $2^{-}$ & 1435.70 & 1435.60 & 1435.60 & 1429.39 & -4.5 & 1429 \\
\hline $3^{+}$ & 1835.47 & 1834.98 & 1834.98 & 1814.24 & & 1830 \\
\hline $3^{-}$ & 2115.41 & 211.5 .08 & 2115.07 & 2101.24 & & 2106.60 \\
\hline $4^{+}$ & 2495.39 & 2485.28 & 2485.28 & 2468.57 & & - \\
\hline 4 & 2873.08 & 2867.55 & 2867.55 & 2851.93 & & - \\
\hline
\end{tabular}

The table also gives these energy differences calculated with the modified form of the effective one-dimensional inversion potential, Eq. $(28 \mathrm{~m})$. The potential constants again follow from those of $\mathrm{NH}_{3}$ and have the values: $\mu_{0}=21.80$ $\mathrm{cm}^{-1} ; F_{0}^{\prime}=1947.81 \mathrm{~cm}^{-1} ; G_{0}^{\prime}=1284.05 \mathrm{~cm}^{-1} ; D=37.80 \mathrm{~cm}^{-1}$. An exact calculation of the perturbation due to $H_{\mathrm{vib}}^{(1)}$ has not been performed and might be difficult to interpret since there exist a number of resonances. However, on the basis of the $H_{\mathrm{vib}}^{(1)}$ contributions for $\mathrm{NH}_{3}$, a very rough estimate has been made of their probable values for $\mathrm{ND}_{3}$. These appear in the next to last column under the heading Estimated Perturbation Correction.

\section{INVERSION-ROTATION SPLITTINGS IN $\mathrm{NH}_{3}$}

The observed infrared spectrum of ammonia shows the rotational fine structure which is characteristic of a symmetric top molecule where, however, the effective rotational constants are functions of the vibrational quantum numbers and of the symmetry $(+$ or - ) of the inversion states. The inversion splitting may therefore be expressed as a function of the rotational quantum numbers through a formula of the form

$$
\Delta_{n}=\Delta_{n}{ }^{0}+\left(B_{n}{ }^{-}-B_{n}{ }^{+}\right)\left[J(J+1)-K^{2}\right]+\left(C_{n}^{-}-C_{n}{ }^{+}\right) K^{2}+\cdots .
$$

Although terms quartic in $J$ and $K$ are often added to this type of expression in order to obtain a more exact fit to the experimentally observed splittings, the dominant contribution comes from the quadratic terms. In the present investigation no attempt will be made to calculate these higher order terms. One of the consequences of the potential function chosen to represent the ammonia molecule is that the potential becomes exceedingly high and the wave function 
vanishingly small for all values of the coordinates for which the configuration departs significantly from pyramidal symmetry. For this reason the total Hamiltonian which includes both rotation and vibration may be represented to first order as the sum of the vibrational Hamiltonian already treated and a rotatiomal Hamiltonian characterizing a symmetrical top. Thus

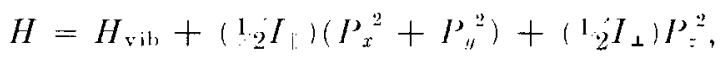

where $I_{\perp}$ is the moment of inertia about the symmetry axis and $I$ is the moment of inertia about an axis perpendicular to the symmetry axis. As usual $P_{x}, P_{y,}$, and $P_{z}$ denote the components of the total angular momentium along the prinripal axes of the molecule.

From Table I it can be seen that the rotation-inversion constants $B_{n}{ }^{--}-B_{n}{ }^{+}$ and $C_{n}{ }^{-}-C_{n}{ }^{+}$are very sensitive functions of the inversion cuantum number, n., while their dependence on the remaining quantum numbers is much less pronounced. This fact suggested the following plan of attack. The Hamiltonian of E(1. (35) was initially simplified by including in $H_{\text {vin }}$ and in $I$ and $I_{\perp}$ only those terms which depend upon the inversion coordinate $x$. The inversion splittings can be calculated with some exactness using perturbation theory. Subreepuently the dependence of $B_{n}{ }^{-}-B_{n}{ }^{+}$and $C_{n}{ }^{-}-C_{n}{ }^{+}$on the remaining roordinates will be examined.

for the purpose of computation it is convenient to rewrite Ece. (35) as

$$
H=H^{(0)}+H^{(1)}
$$

whor"

$$
\begin{aligned}
& H^{(0)} h c=H_{\mathrm{vi} b}^{(1)} h c+B_{c}\left[\mathrm{~J}^{2}-J_{z}^{2}\right]+C_{z} J^{2}, \\
& H^{(1)} h c=H_{\mathrm{vih}}^{(1)} h c+\left(B-B_{c}\right)\left[\mathrm{J}^{2}-J_{z}^{2}\right]+\left(C-C^{2}\right) J_{z}{ }^{2},
\end{aligned}
$$

and

$$
B=h / 8 \pi^{2} c I_{1}, \quad r^{r}=h / 8 \pi^{2} \cdot I_{\perp} .
$$

The rotational coeffieients $B$ and $(*$ are functions of the inversion roordinate whereas $B_{o}=9.965 \mathrm{~cm}^{-1}$ and $c_{r}=6.341 \mathrm{~cm}^{-1}$ are their equilibrium values as estimated by Benedict and Plyler (2). For convenience, dimensionless angular momentum operators $J_{\alpha}=P_{\alpha /} \hbar(\alpha=x, y, z)$ have been introduced. $H_{v i l}^{(i)}$ is given by Eic. (21) and $H_{\mathrm{vih}}^{(1)}$ by $\mathrm{Eq}$. (22).

If the degenerate frequencies are not excited the rotational constants are given by

$$
\begin{aligned}
& B-B_{r}=B_{f}\left[\frac{\sin ^{2} \zeta_{0}-\sin ^{2}(L x)}{\cosh ^{2}} \frac{\left.\sigma_{0}-1\right) /\left(\cosh ^{2}\right.}{\left.\sigma_{0}+1\right)+\sin ^{2}(L x)}\right], \\
& C-C_{e}=C_{e}\left[\cos ^{2} \frac{\zeta_{0}-\cos ^{2}(L x)}{\cos ^{2}(L x)}\right],
\end{aligned}
$$


where $x=\zeta / L$ is the inversion coordinate and $\sigma_{0}$ is the equilibrium value of the coordinate $\sigma$.

The zeroth order Schrödinger equation has solutions

$$
\begin{gathered}
\Psi^{(0)}=\Psi_{\mathrm{vi} h}^{(0)} \psi_{J K M}, \\
E^{(0)}=E_{\mathrm{vib}}^{(0)}+B_{e}\left[J(J+1)-K^{2}\right]+C_{e} K^{2},
\end{gathered}
$$

where $\psi_{J_{K M}}$ is a symmetric top wave function, $\Psi_{\mathrm{vib}}^{(1)}$ is the wave function given by Eq. (27), and $E_{\mathrm{vib}}^{(0)}$ the energy given by Eq. (26). Since the calculation of the vibration-rotation energies will be restricted to terms at most quadratio in the rotational quantum numbers $J$ and $K$, the corrected energies are found to have the form

$$
E=E_{\mathrm{vib}}+B\left(n_{1} n_{2}{ }^{ \pm} n_{3} n_{4}\right)\left[J(J+1)-K^{2}\right]+C\left(n_{1} n_{2}{ }^{ \pm} n_{3} n_{4}\right) K^{2},
$$

where $E_{\mathrm{vib}}$ is the vibrational energy calculated previously.

The quantities of interest in this investigation are the differences of the rotational constants of the $(+)$ and $(-)$ levels, namely,

$$
\begin{aligned}
& B_{n}{ }^{-}-B_{n}{ }^{+}=B\left(n_{1} n_{2}{ }^{-} n_{3} n_{4}\right)-B\left(n_{1} n_{2}{ }^{+} n_{3} n_{4}\right), \\
& C_{n}{ }^{-}-C_{n}{ }^{+}=C\left(n_{1} n_{2}{ }^{-} n_{3} n_{4}\right)-C^{+}\left(n_{1} n_{2}{ }^{+} n_{3} n_{4}\right) .
\end{aligned}
$$

The calculated values of $B_{n}{ }^{-}-B_{n}{ }^{+}$and $C_{n}{ }^{-}-C_{n}{ }^{+}$, for the levels $\left(0, n_{2}{ }^{ \pm}, 0^{0}\right.$, $\left.0^{0}, J, K\right)$ are compared with the observed values in Table XI. The numbers in Table XI are differences of the diagonal matrix elements of $B-B_{e}$ and $C-C_{e}$ when only the inversion coordinate $x$ is considered. As stated, it is believed that these represent the major contributions to the rotational part of the inversion splitting. A comparison with the observations tends to verify this conclusion since the agreement is relatively good. In particular it is gratifying to see that the increase as $n_{2}$ takes on the values $0,1,2$ and the decrease for $n_{2}=3$ is correctly predicted. Although the agreement is quite good it is far from perfect as might be expected from the approximate nature of the calculation. It may be remarked that Sheng et al. (8) and Hadley and Dennison (9) made similar calculations for $n_{2}=0$ and 1 using essentially the same techniques. A compari-

TABLE XI

InVersion-Rotatiox SplitTing in the Levles $\left(0 n_{2} \pm 0^{0} 0^{0} J \quad K\right)$

\begin{tabular}{lllll}
\hline$n_{2}^{ \pm}$ & $\left(B^{-}-B^{+}\right)_{\mathrm{calc}}{ }^{\mathrm{a}}$ & \multicolumn{1}{c}{$\left(B^{+}-B^{+}\right)_{\mathrm{obs}}$} & $\left(C^{-}-C^{+}\right)_{\mathrm{calc}}{ }^{\mathrm{t}}$ & \multicolumn{1}{c}{$\left(C^{-}-C^{+}\right)_{\mathrm{obs}}$} \\
\hline $0^{ \pm}$ & $-0.0055\left(\mathrm{~cm}^{-1}\right)$ & $-0.005054\left(\mathrm{~cm}^{-1}\right)$ & $0.0015\left(\mathrm{~cm}^{-1}\right)$ & $0.001998\left(\mathrm{~cm}^{-1}\right)$ \\
$1^{ \pm}$ & -0.17 & -0.1817 & 0.049 & 0.0721 \\
$2^{ \pm}$ & -0.57 & -0.535 & 0.17 & 0.231 \\
$3^{ \pm}$ & -0.25 & -0.3041 & 0.095 & 0.1034 \\
\hline
\end{tabular}

a Major contributions only, as given by the simple Hamiltonian (35). 
son shows their results to be in substantial agreement with ours revealing the same type of deviations from the experimental data.

Although the differences of the rotational constants depend mainly on the inversion quantum number, $n_{2}$, it is clear that they are also functions of the vibrational fuantum numers $n_{1}, n_{3}$, and $n_{4}$. In order to discuss this dependence it is convernient to write

$$
B\left(n_{1} n_{2} n_{3} n_{4}\right)-B\left(n_{1} n_{2}{ }^{+} n_{3} n_{4}\right)=B\left(0 n_{2}{ }^{-} 00\right)-B\left(0 n_{2}{ }^{+} 00\right)+\Delta B\left(n_{1} n_{2} n_{3} n_{4}\right)
$$

and

$$
C^{\prime}\left(n_{1} n_{2} \cdot n_{3} n_{4}\right)-C^{\prime}\left(n_{1} n_{2}{ }^{+} n_{3} n_{4}\right)=C^{\prime}\left(0 n_{2}{ }^{\prime} 00\right)-C^{\prime}\left(0 n_{2}{ }^{\prime} 00\right)+\Delta C^{\prime}\left(n_{1} n_{2} n_{3} n_{4}\right) .
$$

In the present approximation, $\Delta B$ and $\Delta($ arise from eross terms between offdiagonal matrix elements of $H_{\mathrm{vib}}^{(1)}$ and off-diagonal matrix elements of $B-B_{c}$ and $C^{\prime}-C_{e}$. The calculated values of $\Delta B$ and $\Delta C$ for levels with $n_{2}=1$ arc rompared with the observed values in Table XII. It is seen from this table that the ehanges in $B_{n}{ }^{-}-B_{n}{ }^{+}$and $C^{+}{ }^{-}-C^{+}{ }^{+}$, for levels with $n_{2}=1$, as a function of $n_{1}, n_{3}$, and $n_{4}$, are given surprisingly well by the simple Hamiltonian ' $\mathrm{X}$ pressed hy Ha. (35). In all cases the signs of $\Delta B$ and $\Delta C$ are correct and, with the exception of the level $\left(0,1^{ \pm}, 0^{\prime \prime}, 1^{1}\right)$, the calculated magnitudes agree rather well with the observed numbers. The corresponding numbers for levels with $n_{2}=0$ are shown in Table XIII. The calculated values of $\Delta B$ and $\Delta r^{\prime}$ for thr lesels $\left(1,0^{ \pm}, 0^{0}, 0^{0}\right)$ undoubtedly should be disregarded sines they are strongly dependent on the potential constants $F_{1}$ and $F_{1}$ which were found earlier to be inadequate for describing the inversion-vibration splitting of the pure vibra-

TABLE: XII

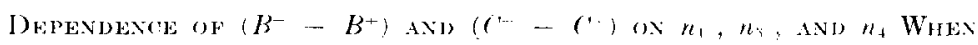

\begin{tabular}{|c|c|c|c|c|}
\hline Level & $\Delta B_{\text {r:ale }}\left(\mathrm{cm}^{-1}\right)^{: 1}$ & 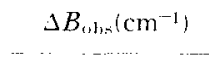 & $\Delta\left(c_{\text {ralc }}\left(\mathrm{cm}^{-1}\right)^{\mathrm{a}}\right.$ & $\Delta C^{\prime} \ln \left(\mathrm{cm}^{-1}\right)$ \\
\hline $11 \cdot 0^{n}\left(0^{\prime \prime}\right.$ & 0.067 & 0.0552 & -0.019 & -0.0251 \\
\hline () $1: 1^{1}\left(0^{\prime \prime}\right.$ & 0.091 & 0.0833 & -0.025 & -0.0292 \\
\hline $01+0011$ & -0.027 & -0.009 & 0.008 & 0.025 \\
\hline $01^{-} 1^{1} 1^{1}$ & 0.064 & 0.052 & -0.018 & -0.018 \\
\hline
\end{tabular}

$$
n:=1
$$

"Major contributions only, as given by the simple Hamiltonian (35).

TABISE XIII

\begin{tabular}{|c|c|c|c|c|}
\hline Level & $\Delta B_{\text {calc }}\left(\mathrm{cm}^{-1}\right)^{*}$ & $\Delta B_{0 \mathrm{hx}}\left(\mathrm{cm}^{-1}\right)$ & $\Delta C_{m_{1} l_{1} \cdot}\left(\mathrm{cm}^{-1}\right)^{*}$ & $\Delta C_{1,1 \times x}\left(\mathrm{~cm}^{1}\right)$ \\
\hline $10^{ \pm}=0^{0} 0^{01}$ & 0.0049 & -0.0007 & -0.0019 & $0.0(0) 1$ \\
\hline $00 \div 1^{1} 0^{0}$ & 0.0041 & 0.0015 & -0.0011 & $-0.0(013$ \\
\hline () $\quad 0^{ \pm}=\left(0^{0} 1^{1}\right.$ & -0.0012 & 0.099 & 0.0003 & 0.009 \\
\hline
\end{tabular}

DEPENDENCE OF $\left(B^{-}-B^{+}\right)$ANI $\left(r^{-}-C^{+}\right)$OX $n_{1}$. n:i , AXD $n_{+}$WHEX $n_{n}=0$

"Major contributions only, as given by the simple Hamiltonian (35). 
tional levels $\left(1,0^{ \pm}, 0^{0}, 0^{0}\right)$. For the levels $\left(0,0^{ \pm}, 1^{1}, 0^{0}\right)$ the calculated and observed values of $\Delta C$ agree well while the corresponding values of $\Delta B$ agree in sign and only roughly in magnitude. The calculated and observed numbers for the levels $\left(0,0^{ \pm}, 0^{0}, 1^{1}\right)$ are not in good agreement and indeed even the sign of $\Delta B$ is not given correctly.

A complete agreement between the calculated and observed rotation-inversion constants should not be expected on the basis of a Hamiltonian as incomplete as Eq. (35). The fact that the agreement is as good as it is appears to verify the assumption that Eq. (35) does indeed contain the dominant terms. An attempt was made to introduce corrections to Eq. (35) for the purpose of examining the next order of approximation. Details of this attempt are given elsewhere $(11)^{3}$ Unfortunately a number of difficulties appeared which prevented any substantial progress being made. In the first place the additional terms which are quite complex in form, contribute mainly through off-diagonal elements and thus second order perturbation theory must be employed. There were found to exist a number of near resonances among the interacting levels which appear to limit the validity of nondegenerate perturbation theory. While the numerical values for these correction terms did not substantially improve the agreement between the calculated and observed rotation-inversion constants, the theory did predict that the fine structure of the resonance perturbed levels $\left(0,0^{ \pm}, 0^{0}, 1^{1}\right)$ and $\left(0,1^{ \pm}, 0^{0}, 1^{1}\right)$ should exhibit marked perturbations of the rotation lines. Experimentally $(3-5)$ it is found that the rotation-inversion pattern for these bands is indeed highly irregular and can not well be expressed by a power series in the rotational quantum numbers. It seems probable that further progress may require important modifications of the inversion potential function as well as refinements in the method of calculation.

Received: March 24, 1961

\section{REFERENCES}

1. W. S. Benedict, E. K. Plyler, and E. D. Tidwell, J. Research Natl. Bur. Standards 61, 123 (1958).

2. W. S. Benedict and E. K. Plyler, Can. J. Phys. 35, 1235 (1957).

9. W. S. Benedict, E. K. Ply iler, and E. D. Tidwell, J. Chem. Phys. 29, 829 (1958).

4. W. S. Benedict, E. K. Plyler, and E. D. Tivwell, J. Chem. Phys. 32, 32 (1960).

5. J. S. Garing, H. H. Nielsen, and K. Narahari Rao, J. Mol. Spectroscopy 3, 496 (1959).

6. D. M. Dennison and G. E. Uhlenbeck, Phys. Rev. 41, 313 (1932).

7. M. F. Manning, J. Chem. Phys. 3, 136 (1935).

8. H. Y. Sheng, E. F. Barker, And D. M. Dennison, Phys, Rev. 60, 786 (1941).

9. L. N. Hadley and D. M. Dennison, Phys. Rev. 70, 780 (1946).

10. D. M. Dennison, Revs. Modern Phys. 12, 175 (1940).

11. W. T. WeEKs, thesis, 1960, University of Michigan (unpublished).

${ }^{3}$ In Ref. (11) an attempt has been made to generalize the complete vibration-rotation Hamiltonian to include the effects of the inversion, and all contributions to the inversionrotation constants of order $B^{2} / \omega$ have been examined. 